\title{
Comparison of Isomerase and Weimberg Pathway for $\gamma$-PGA Production From Xylose by Engineered Bacillus subtilis
}

\author{
Birthe Halmschlag ${ }^{1}$, Kyra Hoffmann ${ }^{2}$, René Hanke ${ }^{2}$, Sastia P. Putri ${ }^{3}$, Eiichiro Fukusaki ${ }^{3}$, \\ Jochen Büchs ${ }^{2}$ and Lars M. Blank ${ }^{1 *}$ \\ ${ }^{1}$ Institute of Applied Microbiology, Aachen Biology and Biotechnology, RWTH Aachen University, Aachen, Germany, \\ ${ }^{2}$ AVT-Biochemical Engineering, RWTH Aachen University, Aachen, Germany, ${ }^{3}$ Department of Biotechnology, Graduate \\ School of Engineering, Osaka University, Osaka, Japan
}

OPEN ACCESS

Edited by:

Ken'ichiro Matsumoto,

Hokkaido University, Japan

Reviewed by:

Mingfeng Cao,

University of Illinois at Urbana-Champaign, United States

Yasushi Ogasawara Hokkaido University, Japan

*Correspondence:

Lars M. Blank

lars.blank@rwth-aachen.de

Specialty section:

This article was submitted to

Biomaterials,

a section of the journa Frontiers in Bioengineering and Biotechnology

Received: 07 October 2019 Accepted: 23 December 2019

Published: 21 January 2020

Citation:

Halmschlag B, Hoffmann K, Hanke R, Putri SP, Fukusaki E, Büchs J and

Blank LM (2020) Comparison of Isomerase and Weimberg Pathway for $\gamma$-PGA Production From Xylose by

Engineered Bacillus subtilis. Front. Bioeng. Biotechnol. 7:476 doi: 10.3389/fbioe.2019.00476
The production of poly- $\gamma$-glutamic acid ( $\gamma$-PGA), a biopolymer consisting of D- and L-glutamic acid monomers, currently relies on L-glutamate, or citrate as carbon substrates. Here we aimed at using plant biomass-derived substrates such as xylose. $\gamma$-PGA producing microorganisms including Bacillus subtilis natively metabolize xylose via the isomerase pathway. The Weimberg pathway, a xylose utilization pathway first described for Caulobacter crescentus, offers a carbon-efficient alternative converting xylose to 2-oxoglutarate without carbon loss. We engineered a recombinant $B$. subtilis strain that was able to grow on xylose with a growth rate of $0.43 \mathrm{~h}^{-1}$ using a recombinant Weimberg pathway. Although ion-pair reversed-phase LC/MS/MS metabolome analysis revealed lower concentrations of $\gamma$-PGA precursors such as 2-oxoglutarate, the $\gamma$-PGA titer was increased 6-fold compared to the native xylose isomerase strain. Further metabolome analysis indicates a metabolic bottleneck in the phosphoenolpyruvate-pyruvate-oxaloacetate node causing bi-phasic (diauxic) growth of the recombinant Weimberg strain. Flux balance analysis (FBA) of the $\gamma$-PGA producing $B$. subtilis indicated that a maximal theoretical $\gamma$-PGA yield is achieved on D-xylose/ $D$-glucose mixtures. The results of the $B$. subtilis strain harboring the Weimberg pathway on such D-xylose/ D-glucose mixtures demonstrate indeed resource efficient, high yield $\gamma$-PGA production from biomass-derived substrates.

Keywords: Bacillus subtilis, $\gamma$-PGA, online viscosity measurement, metabolic engineering, weimberg pathway, xylose, metabolome analysis

\section{INTRODUCTION}

Poly- $\gamma$-glutamic acid ( $\gamma$-PGA) is a biopolymer consisting of D- and L-glutamic acid monomers. The monomers are linked via amide linkages between the $\gamma$-carboxyl and the amino group of monomers $\gamma$-PGA is a non-toxic, biodegradable polymer. Due to these properties, $\gamma$-PGA is suitable for various applications in industrial fields including bioremediation, food sector, and medical use. The chemical synthesis of $\gamma$-PGA is complex since glutamate has two carboxyl groups. Thus, industrial production of this polymer is solely based on bacterial fermentation. The cost of production is hindering many applications of biopolymers (Kreyenschulte et al., 2014). Therefore, 
lowering the production cost by utilization of cheap substrates derived from plant biomass becomes an important task for a more efficient bioprocess of $\gamma$-PGA. One of the suitable substrates is xylose, the second most abundant carbohydrate in nature and the main pentose of hemicellulose in plant biomass. Depending on the origin of the hemicellulose, xylose makes up $90 \%$ of the hemicellulose (Saha, 2003).

The production of $\gamma$-PGA depends on the PGA synthetase catalyzing the polymerization reaction of $\mathrm{L}$ - and $\mathrm{D}$-glutamic acid monomers and exporting the $\gamma$-PGA to the extracellular space. The glutamic acid precursors can be either de novo synthesized or imported from the medium. For de novo synthesis of glutamate from glucose, glucose is converted to acetyl-CoA by glycolysis, which then enters the TCA cycle to form 2-oxoglutarate. The glutamate synthase encoded by glt $A B$ catalyzes the conversion of 2-oxoglutarate and glutamine to L-glutamate (Bohannon et al., 1985; Belitsky et al., 2000). This reaction is NADPH dependent. In absence of glutamine, L-glutamate can be synthesized from 2-oxoglutarate and ammonia by glutamate dehydrogenase. Lglutamate can be converted to D-glutamate directly by glutamic acid racemase or indirectly via D-amino acid aminotransferase (Ashiuchi, 2010).

Besides glucose, B. subtilis 168 can also grow on xylose as sole carbon source. In B. subtilis 168 , xylose is taken up via the arabinose transporter. Xylose is metabolized by a combination of the xylose isomerase pathway and the pentose phosphate pathway (PPP). The genes encoding the xylose isomerase and xylulokinase are $x y l A$ and $x y l B$, respectively. These enzymes convert xylose to xylulose-5-phosphate, which is an intermediate of the PPP. In other bacteria as Pseudomonas taiwanensis VLB120 and Caulobacter crescentus the alternative Weimberg pathway for the conversion of xylose to 2-oxoglutarate has been described (Weimberg, 1961; Stephens et al., 2007; Köhler et al., 2015). The $C$. crescentus $x y l$ operon including the five genes $x y l X A B C D$ was shown to encode the enzymes for this linear $\mathrm{D}$-xylose oxidative pathway. $\mathrm{D}$-xylose is converted via $\mathrm{D}$-xylono-1,4-lactone, xylonate, 2-keto-3-deoxy-D-xylonate, and 2-oxoglutarate-semialdehyde to 2-oxoglutarate (Stephens et al., 2007). The Weimberg pathway was successfully integrated into Pseudomonas putida S12 to enable the utilization of xylose as carbon source (Meijnen et al., 2009). Whereas, for the wild-type strain xylose is catabolized via the PPP, the integration of the Weimberg pathway to B. subtilis and simultaneous deletion of the native xylose degradation pathway likely directs the carbon flux to 2-oxoglutarate with a theoretical carbon yield of $100 \%$ (see Figure 1).

In this study, $\gamma$-PGA production with xylose as substrate is demonstrated. The core stoichiometric model for growth and $\gamma$-PGA formation (Zhu et al., 2013) was extended with metabolic pathways for xylose utilization. The model was used to calculate the $\gamma$-PGA production rate and yield for different substrate compositions. Metabolite measurements were carried out to validate the hypothesized higher precursor supply for xylose metabolism via the Weimberg pathway. Using the engineered strains, $\gamma$-PGA was produced with xylose as sole carbon source as well as with glucose/xylose mixtures. In summary, we present an alternative $\gamma$-PGA production process that can be the basis for future cost efficient and eco-friendly $\gamma$-PGA production.

\section{MATERIALS AND METHODS}

\section{Reagents}

D-glucose, L-glutamic acid, $\mathrm{NH}_{4} \mathrm{Cl}, \mathrm{K}_{2} \mathrm{HPO}_{4}, \mathrm{MgSO}_{4} \cdot 7 \mathrm{H}_{2} \mathrm{O}$, $\mathrm{CaCl}_{2} \cdot 2 \mathrm{H}_{2} \mathrm{O}, \mathrm{MnSO}_{4} \cdot \mathrm{H}_{2} \mathrm{O}, \mathrm{FeCl}_{3} \cdot 6 \mathrm{H}_{2} \mathrm{O}, \mathrm{ZnSO}_{4} \cdot 7 \mathrm{H}_{2} \mathrm{O}, \mathrm{Na}_{2}$ EDTA, $\mathrm{CuSO}_{4} \cdot 5 \mathrm{H}_{2} \mathrm{O}$, and $\mathrm{CoCl}_{2} \cdot 6 \mathrm{H}_{2} \mathrm{O}$ were purchased from Carl Roth GmbH+Co. KG (Karlsruhe, Germany). LC/MS-grade ultra-pure water, HPLC-grade chloroform, acetic acid, $\mathrm{H}_{2} \mathrm{SO}_{4}$, and $\mathrm{NH}_{4} \mathrm{HCO}_{3}$ were purchased from Wako Pure Chemical Industries, Ltd. (Osaka, Japan). 10-Camphorsulfonic acid and tributylamine were purchased from SigmaAldrich (MO, USA).

\section{Strains, Plasmids, and Growth Conditions}

The bacterial strains and plasmids that were developed and used in this study are listed in Table 1. All cloning steps were carried out in Escherichia coli $\mathrm{DH} 5 \alpha$. The recombinase positive E. coli strain JM101 was used to obtain plasmids for the transformation of B. subtilis. For plasmid construction and counterselection, all strains were cultivated at $37^{\circ} \mathrm{C}$ in lysogeny broth (LB) medium containing $100 \mu \mathrm{g} \mathrm{mL}^{-1}$ spectinomycin or $0.5 \%(\mathrm{w} / \mathrm{v})$ mannose as needed. For $\gamma$-PGA production and metabolome analysis, the $B$. subtilis strains were cultured in glucose minimal medium. The media compositions are described in detail in following paragraphs.

\section{Plasmid Construction}

The plasmid pBs-17 was constructed to delete $x y l A B$ in B. subtilis. For pBs-17 construction, two target sequences (TS1 and TS2) located upstream and downstream of $x y l A B$ were amplified from $B$. subtilis genomic DNA using primer pairs BS-85/86 and BS-87/88 (primer listed in Supplementary Table 1). The target sequences were integrated into linearized pJOE-8739 by NEBuilder HiFi DNA Assembly as described by the manufacturer (New England Biolabs, Germany). pJOE-8739 was linearized using primers BS-25/26. For integration of $x y l X A C D^{C C}$ and simultaneous deletion of $x y l A B^{B S}$, the previously constructed pBs-17 was linearized with BS-155 and BS-92. The codonoptimized $x y l X A$ and $x y l C D$ genes were cloned into pJET vectors and amplified with primer pairs BS-156/157 and BS$158 / 159$, respectively. $x y l X A$ and $x y l C D$ were assembled with the linearized pBs-17 to obtain plasmid pBs-24. Plasmid pBs19 was constructed to integrate the P43 into the B. subtilis gdh locus. The plasmid was constructed by integration of TS1, promoter P43, and TS2, which were amplified using primer pairs BS-99/100, BS-101/102, and BS-103/104, respectively, into linear pJOE-8739. Plasmid pBs-34 was constructed to integrate $x y l B^{C C}$ under control of the $\mathrm{P} 43$ promoter into the $B$. subtilis $g d h$ locus. For construction of $\mathrm{pBs}-34$, the previously constructed vector pBs-19 containing the TS1 for integration at the $g d h$ site and the P43 promoter was linearized using primers BS-224/BS225. The $x y l B$ gene and TS2 were amplified with BS-226/227 and BS-228/229, respectively. $\gamma$-PGA production was enabled by pBs-43. The native B. subtilis promoter Pxyl was fused with a CcpA binding site building promoter PX43. The PX43 cre site is 


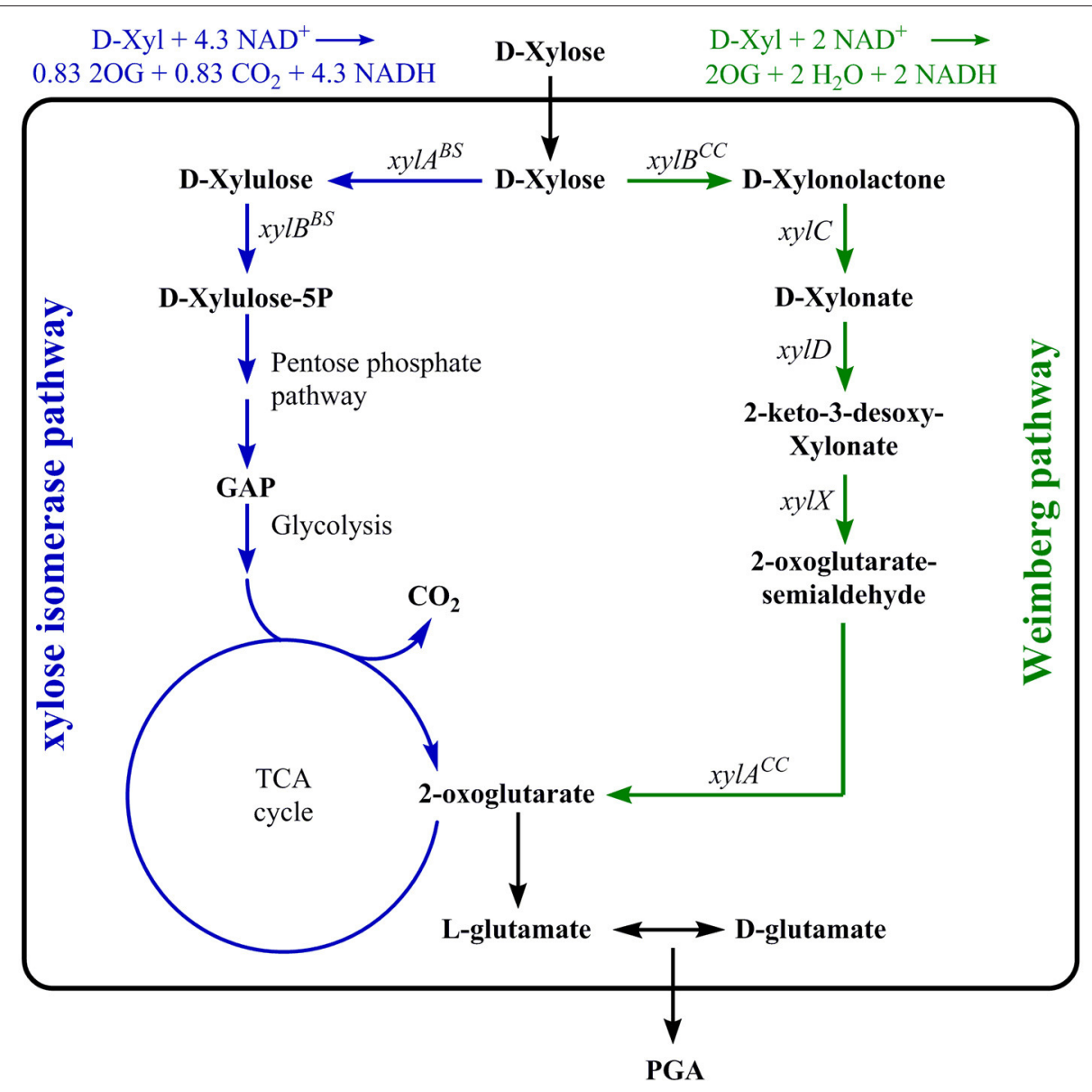

FIGURE 1 | Metabolic pathways for $\gamma$-PGA production with xylose utilization. The isomerase pathway (blue) is natively present in Bacillus subtilis. The genes xylA and $x y I B$ encode the xylose isomerase and xylulokinase, respectively. These enzymes convert xylose to xylulose-5-phosphate, a pentose phosphate pathway (PPP) intermediate. The Weimberg pathway as present in Caulobacter crescentus (green) consists of five genes, $x y / A, x y / B, x y / C, x y / D$, and $x y / X$. It is a linear pathway converting xylose to 2-oxoglutarate.

natively used for expression control of $r b s R$ and $y n c C$ in B. subtilis (Marciniak et al., 2012). The vector contains target sequences for the integration of the promoter upstream of the $p g s$ operon (see Supplementary Information). The assembled plasmids were transformed into chemically competent E. coli DH5 $\alpha$. After analyzing the plasmids by PCR, correctly constructed plasmids were sequenced and retransformed to E. coli JM101 to obtain the plasmids for B. subtilis transformation.

\section{Strain Development}

For the Bacillus transformations, naturally competent cells were prepared according to the Paris method (Harwood, 1990). The $x y l A B$ genes were deleted in $B$. subtilis $\triangle$ spo using the plasmid pBs-17, which resulted in the development of the B. subtilis $\triangle x y l A B$ strain. The integration of the Weimberg pathway was carried out by integration of $x y l X A C D$ into B. subtilis $\Delta$ spo using plasmid pBs-24. The resulting B. subtilis $\triangle x y l A B:: x y l X A C D^{C C}$ strain was transformed with pBs-34 to integrate $x y l B^{C C}$ into the $g d h$ locus. The $\gamma$-PGA production with the native $B$. subtilis pgs operon was enabled by integrating the promoter PX43 resulting in B. subtilis WB PX43-pgs and B. subtilis Ref PX43-pgs for the strain containing the Weimberg pathway and for the reference strain (B. subtilis $\Delta$ spo) that harbors the xylose isomerase pathway, respectively. All genome editing steps were performed using the markerless gene deletion system as described by Wenzel and Altenbuchner (2015).

\section{Flux Balance Model}

The basic model for metabolic reactions required for $\gamma$ PGA synthesis was taken from Zhu et al. (2013). The model represents the central carbon metabolism including glycolysis, TCA cycle, and PPP. The included reaction for biomass formation is derived from Dauner and Sauer (2001). The biomass formation was modeled as a combination of eight precursors (3-phosphoglycerate, phosphoenolpyruvate, ribose-5-phosphate, erythose-4-phosphate, pyruvate, acetyl-CoA, 2-oxoglutarate, and methyl-tetrahydrofolate) and utilization of ATP and NAD $(\mathrm{P}) \mathrm{H}$. The production of $\gamma$-PGA as an ATP-dependent process is included in the model. The previously described metabolic 
TABLE 1 | Strains and plasmids used in this study.

\begin{tabular}{|c|c|c|}
\hline Strain/plasmid & Genotype/properties & Reference/source \\
\hline \multicolumn{3}{|l|}{ STRAINS } \\
\hline $\begin{array}{l}\text { Escherichia coli } \\
\mathrm{DH} 5 \alpha\end{array}$ & $\begin{array}{l}\text { fhuA2 } \Delta \text { (argF-lacZ) U169 phoA } \\
\text { glnV44 Ф80 } \Delta(\text { lacZ)M15 gyrA96 } \\
\text { recA1 relA1 endA1 thi-1 hsdR17 }\end{array}$ & $\begin{array}{l}\text { Meselson and Yuan, } \\
1968\end{array}$ \\
\hline $\begin{array}{l}\text { Escherichia coli } \\
\text { JM101 }\end{array}$ & $\begin{array}{l}\text { glnV44 thi-1 } \Delta \text { (lac-proAB) } \\
\mathrm{F}^{\prime}\left[\text { lacla }^{\prime} Z \Delta M 15 \text { traD36 proAB }{ }^{+}\right]\end{array}$ & Messing et al., 1981 \\
\hline $\begin{array}{l}\text { Bacillus subtilis } \\
\Delta \text { spo }\end{array}$ & $\begin{array}{l}\Delta \mathrm{SP} \beta \Delta \text { skin } \triangle \mathrm{PBSX} \Delta \operatorname{pro} \Phi 1 \\
\Delta p k s:: \mathrm{CmR}, \Delta \text { pro } \Phi 3 \text { trp+ } \\
\Delta m a n P A:: e r m \Delta b p r \Delta \text { sigG } \Delta \text { sigE } \\
\Delta \text { spoGA }\end{array}$ & $\begin{array}{l}\text { Halmschlag et al., } \\
2019\end{array}$ \\
\hline $\begin{array}{l}\text { Bacillus subtilis } \\
\triangle x y \mid A B\end{array}$ & $\Delta$ spo derivative; $\triangle x y \mid A B$ & This study \\
\hline $\begin{array}{l}\text { Bacillus subtilis } \\
\triangle x y \mid A B:: x y I X A C D^{C C}\end{array}$ & $\triangle$ spo derivative; $\triangle x y|A B:: x y| X A C D^{C C}$ & This study \\
\hline Bacillus subtilis WB & $\begin{array}{l}\Delta \text { spo derivative; } \triangle x y|A B:: x y| X A C D^{C C} \\
\Delta \text { gdh::P43-xylB }\end{array}$ & This study \\
\hline $\begin{array}{l}\text { Bacillus subtilis WB } \\
\text { PX43-pgs }\end{array}$ & WB derivative; $\Delta \mathrm{P}(\mathrm{pgs}): \mathrm{PX} 43$ & This study \\
\hline $\begin{array}{l}\text { Bacillus subtilis Ref } \\
\text { PX43-pgs }\end{array}$ & $\Delta$ spo derivative; $\Delta \mathrm{P}($ pgs):: $\mathrm{PX} 43$ & This study \\
\hline \multicolumn{3}{|l|}{ PLASMIDS } \\
\hline pJOE-8739 & Vector backbone & $\begin{array}{l}\text { Wenzel and } \\
\text { Altenbuchner, } 2015\end{array}$ \\
\hline pBs-17 & Deletion of $x y \mid A B$ genes & This study \\
\hline pBs-19 & $\begin{array}{l}\text { Integration of P43 promoter into gdh } \\
\text { locus }\end{array}$ & This study \\
\hline pBs-24 & $\begin{array}{l}\text { Integration of } x y \mid X A C D \text { from } \\
\text { Caulobacter crescentus into } x y I A B \\
\text { locus }\end{array}$ & This study \\
\hline pBs-34 & $\begin{array}{l}\text { Integration of } x y / B \text { from C. crescentus } \\
\text { into gdh locus }\end{array}$ & \\
\hline pBs-43 & $\begin{array}{l}\text { Integration of PX43 promoter } \\
\text { upstream of pgs }\end{array}$ & This study \\
\hline
\end{tabular}

model for $\gamma$-PGA production with B. subtilis (Zhu et al., 2013) was augmented with the two metabolic pathways for xylose utilization. The two reactions of the xylose isomerase pathway, xylose isomerase and xylulokinase were integrated. The heterologous pathway for the conversion of xylose to 2oxoglutarate referred to as Weimberg pathway is added as described by Stephens et al. (2007). The resulting network consists of 48 reactions and 41 metabolites. For the calculation of $\gamma$-PGA production rates, a growth rate of $0.6 \mathrm{~h}^{-1}$ and an ATP maintenance coefficient of $9.9 \mathrm{mmol} \mathrm{g}_{\mathrm{cdw}}^{-1} \mathrm{~h}^{-1}$ as stated for the genome-scale model of B. subtilis by Oh et al. (2007) were used. The substrate uptake rate was fixed to $20 \mathrm{mmol} \mathrm{g}_{\mathrm{cdw}}^{-1} \mathrm{~h}^{-1}$ in total whereas the actual substrate uptake rate and by-product formation rates depend on the exact cultivation conditions and have to be determined. Based on these values, the $\gamma$-PGA production rate was calculated by flux balance analysis (FBA) for different substrate compositions with the $\gamma$-PGA production rate as objective function. Flux balance analysis was carried out using the Cobra Toolbox (Schellenberger et al., 2011) and Matlab (The MathWorks, Inc.).

\section{Cultivation for Metabolite Analysis}

For the cultivation of $B$. subtilis either xylose or xylonate was used as carbon source in a minimal salt medium for the main cultures. The minimal medium contained: $20 \mathrm{~g}$ Xylose, $7 \mathrm{~g} \mathrm{NH}_{4} \mathrm{Cl}, 0.5 \mathrm{~g} \mathrm{KH}_{2} \mathrm{PO}_{4}, 0.5 \mathrm{~g} \mathrm{MgSO}_{4}, 0.2 \mathrm{~g} \mathrm{~L}$-glutamic acid, $0.15 \mathrm{~g} \mathrm{CaCl}_{2}, 0.1 \mathrm{~g} \mathrm{MnSO}_{4}, 0.04 \mathrm{~g} \mathrm{FeCl}_{3}$, and $1 \mathrm{~mL}$ of a trace element solution per liter. The trace element solution [according to Wenzel et al. (2011)] was modified and contained: $0.54 \mathrm{~g}$ $\mathrm{ZnSO}_{4} \cdot 7 \mathrm{H}_{2} \mathrm{O}, 30.15 \mathrm{~g} \mathrm{Na}_{2}$-EDTA, $0.48 \mathrm{~g} \mathrm{CuSO}_{4} \cdot 5 \mathrm{H}_{2} \mathrm{O}$, and $0.54 \mathrm{~g} \mathrm{CoCl}_{2} \cdot 6 \mathrm{H}_{2} \mathrm{O}$ per liter. The medium was buffered using $0.1 \mathrm{M}$ potassium phosphate at $\mathrm{pH}$ 7. To obtain xylonate as carbon source, xylose was dissolved in $0.2 \mathrm{M}$ potassium phosphate buffer $(\mathrm{pH} 7)$ to a concentration of $40 \mathrm{~g} \mathrm{~L}^{-1}$. Pseudomonas putida KT2440, which can convert xylose to xylonate, but is not able to grow on xylose/xylonate, was grown in LB medium overnight. Subsequently, the cells were centrifuged $(5,000 \mathrm{~g}, 10 \mathrm{~min}, \mathrm{RT})$ and added to the xylose solution. The mixture of xylose and cells was incubated for $16 \mathrm{~h}$ to allow the conversion of xylose to xylonate. Afterwards, the cells were removed by centrifugation and the solution was sterile filtered. By addition of the other components, the medium was created as described above with xylonate as C-source instead of xylose. For preculturing, the Bacillus cells were cultivated in LB medium overnight and then transferred to fresh minimal medium containing the same ingredients as the main culture medium. The LB medium contained $10 \mathrm{~g} \mathrm{~L}^{-1}$ tryptone, $5 \mathrm{~g} \mathrm{~L}^{-1}$ yeast extract, and $10 \mathrm{~g} \mathrm{~L}^{-1} \mathrm{NaCl}$ and had a $\mathrm{pH}$ of 7.4. The main cultures were inoculated to an $\mathrm{OD}_{600}$ of 0.1 from the preculture. Except for the trace element solution, which was sterile filtered, all the media components were sterilized by autoclaving for $20 \mathrm{~min}$ at $121^{\circ} \mathrm{C}$. The cultivations were carried out in $250 \mathrm{~mL}$ Erlenmeyer flasks containing $25 \mathrm{~mL}$ medium. The cultures were incubated on a rotary shaker with a $25 \mathrm{~mm}$ shaking diameter at $37^{\circ} \mathrm{C}$ and $200 \mathrm{rpm}$ (Bio-Shaker BR-3000LF, Taitec, Saitama, Japan). All the cultivations were performed in triplicate, and the data are presented as the mean. The cell growth was monitored by $\mathrm{OD}_{600}$ measurements of samples obtained from the shake flasks using the GeneQuant 100 spectrophotometer (GE Healthcare UK Ltd., Buckinghamshire, UK).

\section{Cultivation for On-Line Monitoring (OTR and Viscosity Measurements)}

On-line monitoring of the main cultures was realized using an in-house manufactured Respiration Activity Monitoring System (RAMOS) recording the oxygen transfer rate (OTR) (Anderlei and Büchs, 2001; Anderlei et al., 2004). Commercial versions of this system are available from Kuhner AG, Birsfelden, Switzerland or HiTech Zang, Herzogenrath, Germany.

For main cultures in shake flasks, a master mix was prepared by inoculating the mineral medium with the pre-culture. Subsequently, the desired filling volume was transferred to RAMOS flasks and to additional Erlenmeyer flasks with cotton plugs for sampling and off-line analysis. Main cultures were run under the following conditions: $250 \mathrm{~mL}$ shake flasks, filling volume $20 \mathrm{~mL}$, shaking frequency $200 \mathrm{rpm}$, shaking diameter $50 \mathrm{~mm}$, temperature $37^{\circ} \mathrm{C}$, initial $\mathrm{OD}_{600}=0.1$. RAMOS flasks and sampling flasks were filled from the same master mix and 
cultivated in parallel and under identical conditions to guarantee that cultures ran synchronously. Erlenmeyer flasks withdrawn for sampling were not placed back on the shaker.

For online viscosity measurement a new device according to Sieben et al. (2019) was used. It evaluates the shift in the angular position of the bulk liquid relative to the direction of the centrifugal acceleration. The measurement system is different from the previously used, based on power input measurement (Regestein Née Meissner et al., 2017).

\section{Batch Fermentations}

The batch fermentations were carried out in a stirred tank bioreactor (Eppendorf BioFlo120) equipped with a $\mathrm{pH}$ electrode, dissolved oxygen (DO)-electrode, a temperature sensor, two Rushton turbines, a sampling tube, and a cell growth quantifier for bioreactors (CGQ BioR; Aquila Biolabs, Baesweiler, Germany). The reactor was sterilized at $121^{\circ} \mathrm{C}$ for $20 \mathrm{~min}$ at $1 \mathrm{bar}$ overpressure. The experiments were carried out at a temperature of $37^{\circ} \mathrm{C}$ with a filling volume of $0.5 \mathrm{~L}$. As culture medium, the identical glucose minimal medium as used for the shake flask experiments was employed. The $\mathrm{pH}$ value was adjusted to 7 prior to inoculation and kept constant by the addition of $2 \mathrm{M} \mathrm{HCl}$ or $2 \mathrm{M} \mathrm{NaOH}$ throughout the fermentation. The aeration rate was set to $1 \mathrm{vvm}$. The cultivation was stirred with an agitation rate of $800 \mathrm{rpm}$. Prior to inoculation $0.5 \mathrm{~mL} \mathrm{~L}^{-1}$ antifoam was added to avoid foam formation. At time point $t_{0}$ the reactor was inoculated with cells from a pre-culture to obtain an initial $\mathrm{OD}_{600}$ of 0.1 . For this purpose, the required pre-culture volume was centrifuged $\left(5,000 \mathrm{~g}, 5 \mathrm{~min}, 4^{\circ} \mathrm{C}\right)$, washed with $0.9 \% \mathrm{NaCl}$ and resuspended in $3 \mathrm{~mL} 0.9 \% \mathrm{NaCl}$. The suspension was directly injected into the fermenter.

\section{Analysis of $\gamma$-PGA Concentration}

The concentration of $\gamma$-PGA was determined by the photometric cetyltrimethylammonium bromide (CTAB) assay. CTAB binds to negatively charged molecules, in the case of $\gamma$-PGA forming a water-insoluble complex, resulting in an increased turbidity of the solution. Samples taken from shake flask or bioreactor fermentations were centrifuged $\left(16,000 \mathrm{~g}, 60 \mathrm{~min}, 4^{\circ} \mathrm{C}\right)$. The sample supernatant was stored at $-20^{\circ} \mathrm{C}$ until analyzed. Based on a calibration curve using standards of $1 \mathrm{MDa} \gamma$-PGA in the range of $0.1-10 \mu \mathrm{g} \mathrm{mL}^{-1}$, the $\gamma$-PGA from the culture supernatant was quantified by measuring the turbidity at $400 \mathrm{~nm}$. For this purpose, $100 \mu \mathrm{L} 0.7 \mathrm{M}$ CTAB in $2 \% \mathrm{NaOH}$ were added to $100 \mu \mathrm{L}$ sample or standard solution in a 96-well microtiter plate. After $1 \mathrm{~min}$ of incubation the turbidity was measured in a Synergy MX microplate reader (BioTek Instruments, Winooski, USA).

\section{Sample Preparation for Metabolome Analysis}

A sample volume satisfying the equation: sampling volume $(\mathrm{mL})$ $\times \mathrm{OD}_{600}=5$ was filtered with a PVDF filter (pore size $0.45 \mu \mathrm{m}$ ) using vacuum. The filter was washed with the doubled amount of $300 \mathrm{mM} \mathrm{NH}_{4} \mathrm{HCO}_{3}$ (1:2 ratio, sample: $\mathrm{NH}_{4} \mathrm{HCO}_{3}$ ) solution. Subsequently, the metabolism was quenched by soaking the filter in liquid $\mathrm{N}_{2}$. The filter was transferred to a $2 \mathrm{~mL}$ sample tube and was stored at $-80^{\circ} \mathrm{C}$ until metabolite extraction. To extract the metabolites, $1.875 \mathrm{~mL}$ of extraction solvent $\left(1 \mathrm{H}_{2} \mathrm{O}: 2 \mathrm{MeOH}: 2\right.$ chloroform, including $7 \mathrm{nM}$ 10-camphorsulfonic acid as internal standard) was added to the sample tube including the filter. After vortexing, the sample tube was centrifuged at 16,000 $\mathrm{g}$ for $3 \mathrm{~min}$ at $4{ }^{\circ} \mathrm{C}$. Three hundred fifty microlitre of the supernatant were transferred to a $1.5 \mathrm{~mL}$ sampling tube, concentrated by vacuum centrifugation for $2 \mathrm{~h}$ and freeze dried overnight. The pellet was stored at $-80^{\circ} \mathrm{C}$ until analyzed by LC-MS.

\section{Ion-Pair-LC/MS/MS Analysis}

The dried sample was resuspended in $50 \mu \mathrm{L}$ ultra-pure water and was transferred into a conical glass vial. The ion-pairliquid chromatography coupled with tandem mass spectrometry (LC/MS/MS) analysis was performed using a Shimadzu Nexera UHPLC system coupled with an LCMS 8030 Plus device (Shimadzu Co., Kyoto, Japan). The system was equipped with a PE capped CERI L-column 2ODS column $(2.1 \times 150 \mathrm{~mm}$, particle size $3 \mathrm{~mm}$, Chemicals Evaluation and Research Institute, Tokyo, Japan). As mobile phase a gradient of a mixture of solvent $A$ and $B$ was used, where solvent $A$ is $10 \mathrm{mM}$ tributylamine and $15 \mathrm{mM}$ acetate in ultra-pure water and solvent $\mathrm{B}$ was pure methanol. The flow rate was set to $0.2 \mathrm{~mL} \mathrm{~min}^{-1}$. For gradient elution of metabolites, starting from $0 \%$ concentration of solvent $\mathrm{B}$, the concentration of $\mathrm{B}$ was increased to $15 \%$ after $1 \mathrm{~min}$ with a gradient of $30 \% \mathrm{~min}^{-1}$, hold for $1.5 \mathrm{~min}$, increased to $50 \%$ within $5 \mathrm{~min}$ and subsequently increased to $100 \%$ within $2 \mathrm{~min}$. The $100 \%$ solvent B concentration was held for $1.5 \mathrm{~min}$, decreased to $0 \%$ from $11.5 \mathrm{~min}$ on and held at this concentration for $8.5 \mathrm{~min}$. The column oven temperature was set to $45^{\circ} \mathrm{C}$. The MS parameters were as follows: probe position, $1.5 \mathrm{~mm}$; desolvation line temperature, $250^{\circ} \mathrm{C}$; drying gas flow, $15 \mathrm{~L} / \mathrm{min}$; heat block temperature, $400^{\circ} \mathrm{C}$; and nebulized gas flow, $2 \mathrm{~L} \mathrm{~min}{ }^{-1}$. As blank sample, a filter washed with $\mathrm{NH}_{4} \mathrm{HCO}_{3}$ without biomass addition was used for extraction of compounds that are not of biological origin. As a Quality Control (QC) sample, $2 \mu \mathrm{L}$ of each analyzed sample of a batch were pooled into a vial. For each sample (including the QC sample), $3 \mu \mathrm{L}$ were injected to the ion-pairLC/MS/MS for metabolite analysis.

\section{Data Processing and Analysis}

The calculation of the peak area was carried out using MRMPROBS ver. 2.38 and checked manually. Peaks that are not of biological origin as identified with the blank sample were excluded. The data was normalized according to the peak area of the internal standard, 10-camphorsulfonic acid. SIMCA 13 (Umetrics, Umeå, Sweden) was used for principal component analysis (PCA).

\section{RESULTS}

\section{Metabolic Pathways for Xylose Utilization and Growth of Engineered Strains}

Stoichiometric comparison of the xylose isomerase pathway (Figure 1, blue) and the Weimberg pathway (green) suggested the benefit of the Weimberg pathway for $\gamma$-PGA production using xylose as carbon source. The conversion of xylose to 2oxoglutarate via the Weimberg pathway theoretically results in 
TABLE 2 | Growth of engineered B. subtilis strains on xylose and xylonate.

\begin{tabular}{lcc}
\hline Strain & \multicolumn{2}{c}{ Growth rate $\mathbf{~ h}^{-\mathbf{1}}$ ] } \\
\cline { 2 - 3 } & Xylonate & Xylose \\
\hline Bacillus subtilis $\triangle$ spo & n.d. & $0.22 \pm 0.02$ \\
Bacillus subtilis $\triangle x y I A B$ & no growth & No growth \\
Bacillus subtilis $\triangle x y \mid A B:: x y I X A C D^{C C}$ & $0.47 \pm 0.01$ & No growth \\
Bacillus subtilis WB & n.d. & $0.43 \pm 0.01$ \\
\hline
\end{tabular}

Bacillus subtilis $\Delta$ spo encodes the isomerase pathway. Bacillus subtilis $\triangle x y \mid A B$ was used as negative control without any catabolic pathway for xylose metabolism. Bacillus subtilis WB contains the genes encoding the Weimberg pathway. n.d., not determined.

$1 \mathrm{~mol} 2$-oxoglutarate per mol xylose. On the contrary, the xylose isomerase pathway yields $0.83 \mathrm{~mol} 2$-oxoglutarate per mol xylose due to the formation of $\mathrm{CO}_{2}$ in the TCA cycle. However, the amount of produced redox equivalents is higher if xylose is metabolized via the xylose isomerase pathway.

Based on the stoichiometric comparison, the insertion of the Weimberg pathway is hypothesized to result in higher precursor concentrations for $\gamma$-PGA synthesis. Therefore, the native xylose isomerase pathway encoded by the genes $x y l A B$ coding for the xylose isomerase and xylulokinase, was replaced by the genes $x y l X A B C D$ originating from $C$. crescentus. As a negative control, the deletion mutant $B$. subtilis $\triangle x y l A B$ not capable of growing on xylose was used. To test the effect of the deletion of the $x y l A B$ genes in $B$. subtilis and the subsequent integration of the Weimberg pathway, the strains $B$. subtilis $\Delta$ spo (reference with isomerase pathway), B. subtilis $\triangle x y l A B$ (negative control), B. subtilis $\triangle x y l A B:: x y l X A C D^{\mathrm{CC}}$, and B. subtilis WB $\left(\triangle x y l A B:: x y l X A C D^{C C} \triangle g d h:: x y l B\right)$ were grown on xylose and xylonate as substrates (Table 2).

The initial strain $B$. subtilis $\Delta$ spo is capable of growing on xylose as sole carbon. The growth rate on xylose was $0.22 \mathrm{~h}^{-1}$ without the addition of arabinose. For the laboratory strain B. subtilis 168, the addition of arabinose is reported to be required for the expression of $a r a E$. AraE is a transport protein of the major facilitator superfamily responsible for the uptake of pentoses including both arabinose and xylose. The deletion of $x y l A B$ resulted in the abolishment of growth on xylose as well as on xylonate. Since xylonate was commercially not available as carbon source, it was obtained from xylose conversion by the $P$. taiwanensis VLB120 glucose dehydrogenase. Therefore, the xylonate substrate contained xylose impurities. For this reason the growth rate of the reference strain and the Weimberg mutant was not tested on xylonate. For the mutant harboring the incomplete Weimberg pathway B. subtilis $\triangle x y l A B:: x y l X A C D^{C C}$ the growth rate on xylonate was $0.47 \mathrm{~h}^{-1}$. This strain is not able to grow on xylose as sole carbon source, as it lacks a functional xylose dehydrogenase $(\mathrm{XDH})$ catalyzing the first step of the Weimberg pathway. The XDH converts xylose to xylonolactone. In other bacteria such as $P$. putida, the integration of the four genes $x y l X, x y l A, x y l C$, and $x y l D$ was sufficient to enable growth on xylose, since the inherent glucose dehydrogenase (GDH) also accepts xylose as substrate (Meijnen et al., 2009). However, the B. subtilis GDH does not catalyze the conversion of xylose (Fujita et al., 1977). Therefore, the ability of $B$. subtilis to grow on xylose was only restored in $\triangle x y l A B$ when all five genes of the Weimberg pathway were integrated into the genome. As shown in Table 2, the Weimberg pathway mutant exhibits a significantly higher growth rate $\left(0.43 \mathrm{~h}^{-1}\right)$ than the reference strain $\left(0.22 \mathrm{~h}^{-1}\right)$ on xylose.

\section{Measurement of Intracellular Precursor Supply}

Since xylose is directly converted to 2-oxoglutarate, a higher precursor supply for $\gamma$-PGA production was hypothesized for growth utilizing the Weimberg pathway. For the comparison of the precursor supply in B. subtilis $\mathrm{WB}$ and the reference strain with and without $\gamma$-PGA production, metabolome analysis using ion pair LC/MS/MS was performed. To enable $\gamma$-PGA production on xylose as carbon source, a xylose inducible promoter fused with a catabolite response element (cre) site as it is present in the genome of B. subtilis (Marciniak et al., 2012) were integrated to control the expression of $p g s$ in B. subtilis Ref PX43-pgs and B. subtilis WB PX43-pgs. Ninety eight metabolites, including central metabolites, amino acids, nucleotides, and cofactors, were annotated in the four strains B. subtilis Ref, $B$. subtilis Ref PX43-pgs, B. subtilis WB, and B. subtilis WB PX43-pgs (Supplementary Table 2). The metabolic profiles of these four strains were subjected to principal component analysis (PCA, Figure 2). PCA of the datasets revealed lower concentrations for intermediates of the TCA cycle, especially 2-oxoglutarate for $B$. subtilis $\mathrm{WB}$ in comparison to the reference catabolizing xylose through the xylose isomerase pathway (Figures 2, 3). In contrast the concentrations for intermediates of glycolysis and PPP as represented by high loading values for ribose-1phosphate, D-glyceraldehyde 3-phosphate (GAP), or fructosebisphosphate (FBP) were increased for growth via the Weimberg pathway. The results indicate a higher TCA cycle flux in the Weimberg pathway mutant, which is in accordance with the highly increased growth rate for $B$. subtilis WB. In turn, fluxes in gluconeogenesis and PPP are likely reduced in this strain, resulting in higher concentrations of the intermediates. For the production of $\gamma$-PGA, the utilization of the Weimberg pathway is favorable as the higher flux through the 2-oxoglutarate results in higher $\gamma$-PGA production.

Investigating the growth behavior on xylose via the Weimberg pathway, a plateau indicating diauxic growth was observed for $\gamma$-PGA producing B. subtilis WB PX43-pgs and non-producing $B$. subtilis $\mathrm{WB}$ at optical densities of 5 and 2, respectively (Figure 4). To investigate this phenomenon, the metabolic state of the cells before (1), during (2), and after (3) the observed plateau was examined by metabolome analysis. The obtained metabolome data was analyzed by PCA. The changes in metabolite concentrations are presented by the score and loading plot (Figure 5) and by the metabolic profile shown in Figure 6. The PCA showed a separation of the three growth stages for the first component (PC1). Metabolites with high positive loading values are decreasing from the first to the third growth phase. Especially the concentrations of glycolysis and PPP intermediates decrease throughout the growth phases 


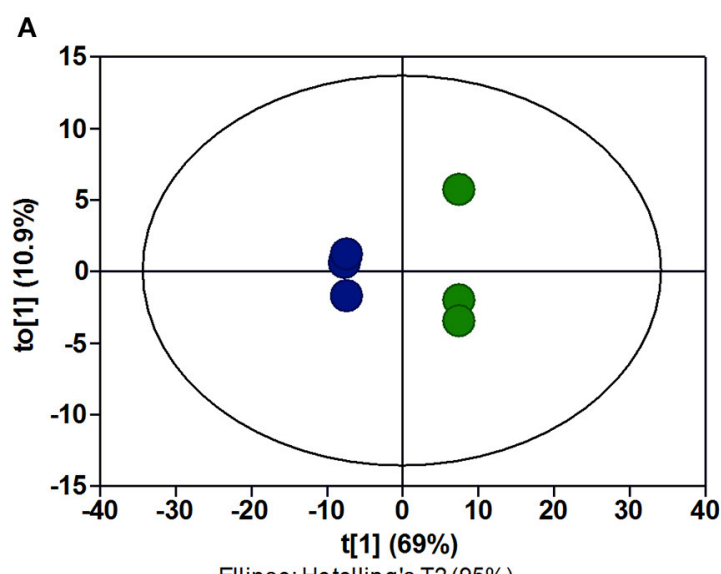

B

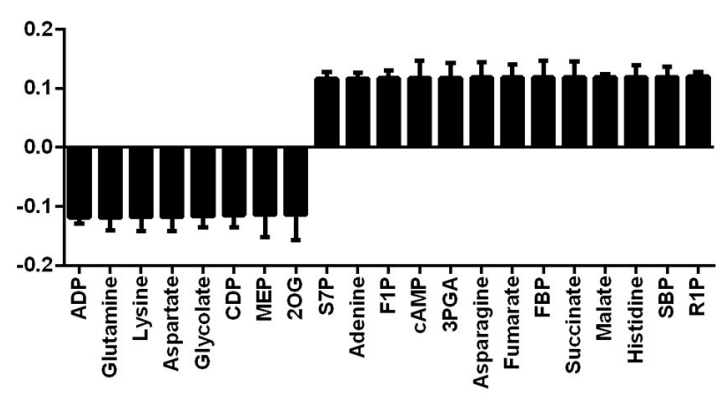

C

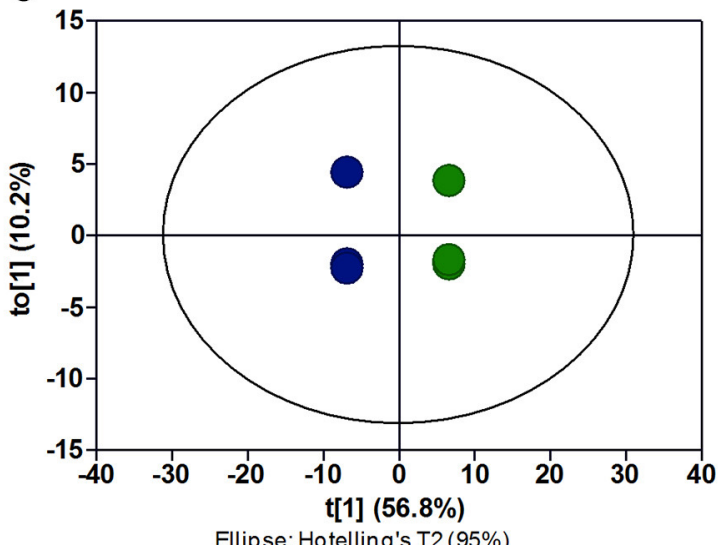

D

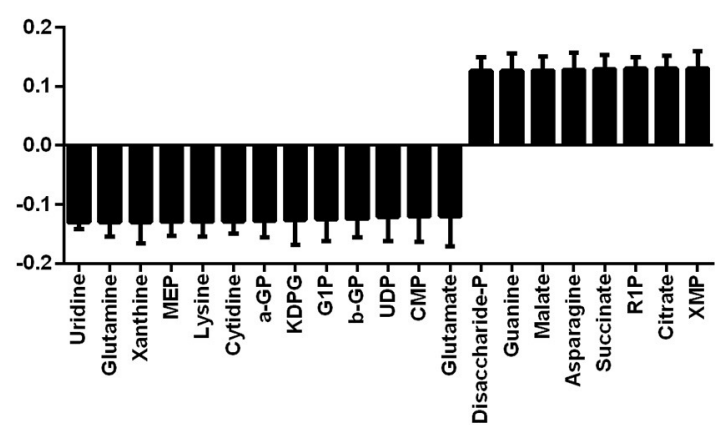

FIGURE 2 | Orthogonal partial least squares discriminant analysis (OPLS-DA) for intracellular metabolites of $B$. subtilis WB in comparison to $B$. subtilis $\triangle$ spo (reference) without (A,B) and with (C,D) $\gamma$-PGA production. The score plot for triplicate metabolome samples of the reference (blue) and $B$. subtilis WB (green) strains without (A) and with (C) $\gamma$-PGA production is given. The loading values without (B) and with (D) $\gamma$-PGA production for metabolites highly contributing to the separation are presented. High loading values correspond to increased metabolite concentrations for B. subtilis WB (PX43-pgs). 2OG, 2-oxoglutarate; 3PGA, 3-Phosphoglycerate; ADP, Adenosine 5'-diphosphate; a-GP, $\alpha$-glycerophosphate; b-GP, $\beta$-glycerophosphate; cAMP, $3^{\prime}, 5^{\prime}$-cyclic AMP; CDP, cytidine 5'-diphosphate; CMP, cytidine 5'-monophosphate; CTP, cytidine 5'-triphosphate; DHAP, dihydroxyacetone phosphate; F1P, fructose-1-phosphate; FBP, fructose-bisphosphate; G1P, glucose-1-phosphate; GAP, D-glyceraldehyde 3-phosphate; GDP, guanosine 5'-diphosphate; KDPG, 2-keto-3-deoxy-6-phosphogluconate; MEP, methyerythritol 4-phosphate; PEP, phosphoenolpyruvate; R1P, ribose-1-phosphate; R5P, ribose-5-phosphate; S7P, sedoheptulose-7-phosphate; SBP,

sedoheptulose-1,7-bisphosphate; UDP, uridine 5'-diphosphate; XMP, xanthosine 5'-phosphate.

as indicated by high loading values for ribulose-5-phosphate, sedoheptulose-7-phosphate (S7P), FBP, and GAP. In contrast, glycolate and glycerate are increasing. These two metabolites are formed in several metabolic pathways such as the glyoxylate shunt or serine metabolism. Further, these two metabolites have been shown to be formed in the ethylene glycol degradation pathway (Antoniewicz, 2015). But none of these pathways is assigned to $B$. subtilis. Regarding the observed plateau, the metabolome data reveals that several metabolites from TCA cycle (succinate, fumarate, and malate) accumulate during the second phase that represents the lag phase (Figure 6). In contrast, pyruvate is clearly decreased during the plateau phase. This indicates that the bottleneck reaction hindering the growth in that phase seems to be the provision of pyruvate. Recently, labeling experiments with a switch from ${ }^{13} \mathrm{C}$-glucose to ${ }^{12} \mathrm{C}$ malate as carbon source demonstrated the inability of $B$. subtilis to instantaneously metabolize malate into the gluconeogenic direction (van Gulik et al., 2000). The delay in gluconeogenic malate metabolism is presumed to be due to the $\mathrm{CcpN}$ repression of the PEP carboxykinase (PckA). The regulation of the B. subtilis metabolism growing on xylose via the Weimberg pathway has to be further investigated.

\section{$\gamma$-PGA Production via Weimberg Pathway}

To enable the decoupling of growth and production phase for mixed substrates, the combination of xylose-inducible and glucose-repressed promoter elements present in promoter PX43 was chosen. The decoupling of cell growth and $\gamma$-PGA production may be beneficial for a higher biocatalyst yield in the growth phase on glucose and an induced expression of the PGA synthetase after glucose is depleted. The promoter was integrated upstream of $p g s$ in the strain background of the reference and recombinant Weimberg pathway strains. The strains were tested according to their growth behavior and $\gamma$-PGA production in batch fermentations with xylose as carbon source (Figure 7).

As in the shake flask experiments for the non- $\gamma$-PGA producing strains, the Weimberg pathway sustained with $0.37 \mathrm{~h}^{-1}$ a higher growth rate than observed for the reference 


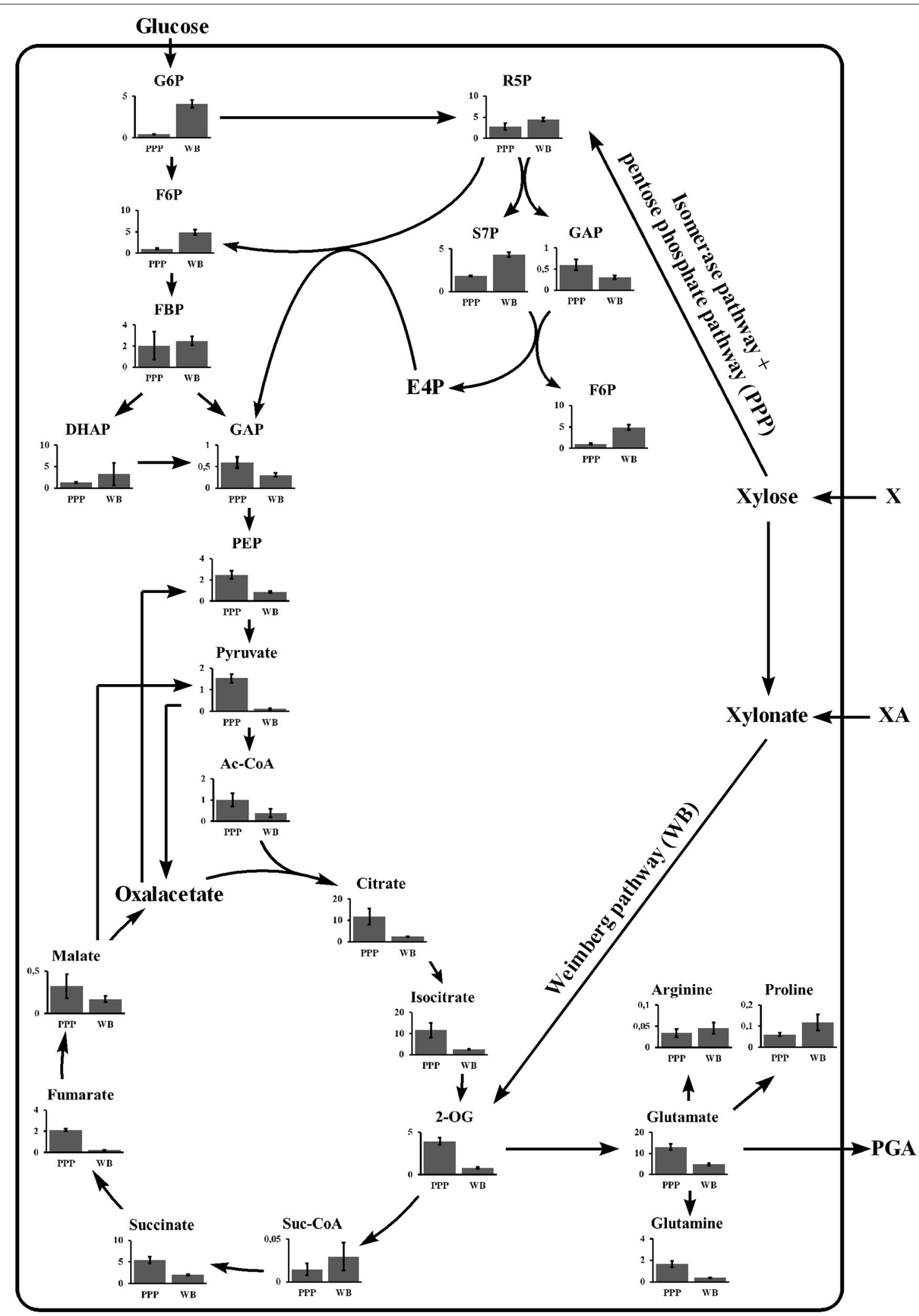

FIGURE 3 | Intracellular metabolite analysis for B. subtilis WB and B. subtilis $\triangle$ spo growing on xylose. The metabolite concentrations for glycolysis, PPP, TCA cycle, and glutamate derived amino acids are given for $B$. subtilis $\triangle \mathrm{spo}$ (PPP) and B. subtilis WB (WB). Cultivations were carried out in triplicates. The bar graphs represent the mean. The error bar indicates the standard deviation. 2OG, 2-oxoglutarate; DHAP, dihydroxyacetone phosphate; F6P, fructose-6-phosphate; FBP,

fructose-bisphosphate; G6P, glucose-6-phosphate; GAP, D-glyceraldehyde 3-phosphate; R5P, ribose-5-phosphate; S7P, sedoheptulose-7-phosphate. 
strain $\left(0.28 \mathrm{~h}^{-1}\right)$. Consistently with the shake flask cultivations, the growth curve of $B$. subtilis WB PX43-pgs shows a plateau for $\sim 1 \mathrm{~h}$ where the biomass concentration is not increasing. Further, B. subtilis WB PX43-pgs exhibits a shorter lag phase than the reference strain. The process time for $B$. subtilis WB PX43-pgs is shorter resulting in a higher $\gamma$-PGA production rate. For B. subtilis WB PX43-pgs, also the maximal obtained $\gamma$-PGA concentration was with $1.05 \mathrm{~g} \mathrm{~L}^{-1}$ higher than the $0.18 \mathrm{~g} \mathrm{~L}^{-1} \gamma$ PGA for the reference. The obtained titer equals to yields of 0.06

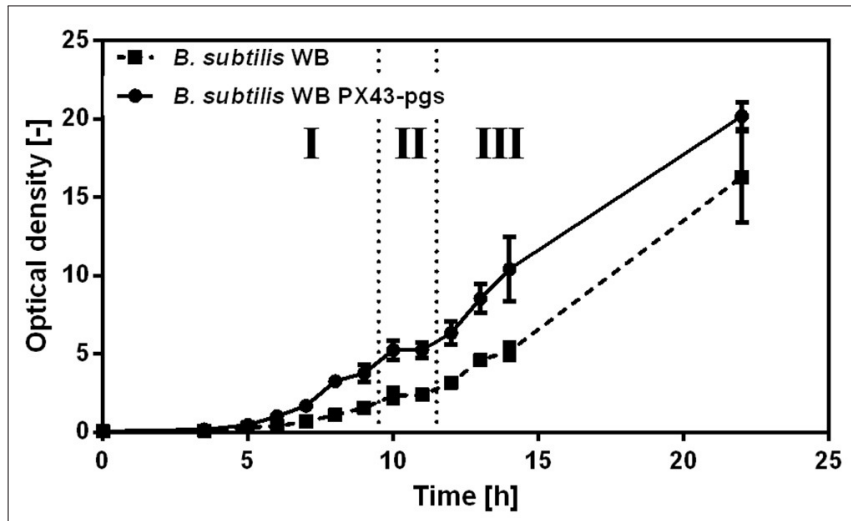

FIGURE 4 | Growth of B. subtilis WB on xylose. Samples for metabolome analysis were taken in the three indicated growth phases. The plateau was observed for B. subtilis WB and B. subtilis WB PX43-pgs, thus independent from $\gamma$-PGA production. Cultivations were carried out in triplicates. Data represents the mean of biological triplicates. The error bar indicates the standard deviation. and $0.01 \mathrm{C}$-mol $\gamma$-PGA per C-mol xylose for the recombinant Weimberg strain and the reference strain, respectively. Based on the results for the intracellular metabolite analysis, the higher $\gamma$-PGA production for the strain using the Weimberg pathway is likely caused by higher flux through the 2-oxoglutarate knot. Thereby a higher rate for the supply of glutamate is possible. However, the obtained yields are much lower than the theoretical yields for $\gamma$-PGA. To further investigate the theoretical flux distributions, the $\gamma$-PGA production was modeled using a stoichiometric model and FBA.

\section{Modeling of $\gamma$-PGA Production for Alternative Growth Conditions}

The core metabolic model for $\gamma$-PGA-producing B. subtilis includes the central carbon metabolism, biomass formation, ATP maintenance, by-product formation and $\gamma$-PGA production. Based on the stoichiometric model, the theoretical C-mol yield for substrate mixtures containing xylose and glucose were calculated. For the calculation of $\gamma$-PGA production rates, a growth rate of $0.6 \mathrm{~h}^{-1}$ and an ATP maintenance coefficient of $9.9 \mathrm{mmol} \mathrm{g}_{\mathrm{cdw}}^{-1} \mathrm{~h}^{-1}$ were used as previously published for the genome-scale model of $B$. subtilis by Oh et al. (2007). Since the $\gamma$ PGA biosynthesis is an ATP-dependent reaction, the theoretical flux distribution is directly influenced by the chosen value for the ATP maintenance coefficient. The substrate uptake rate was fixed to $20 \mathrm{mmol} \mathrm{g}_{\mathrm{cdw}}^{-1} \mathrm{~h}^{-1}$, whereas the actual substrate uptake rate rates vary for the examined cultivation conditions. Based on these values, the $\gamma$-PGA production rate was calculated by FBA for different substrate compositions with the $\gamma$-PGA production rate as objective function. This approach enabled the theoretical
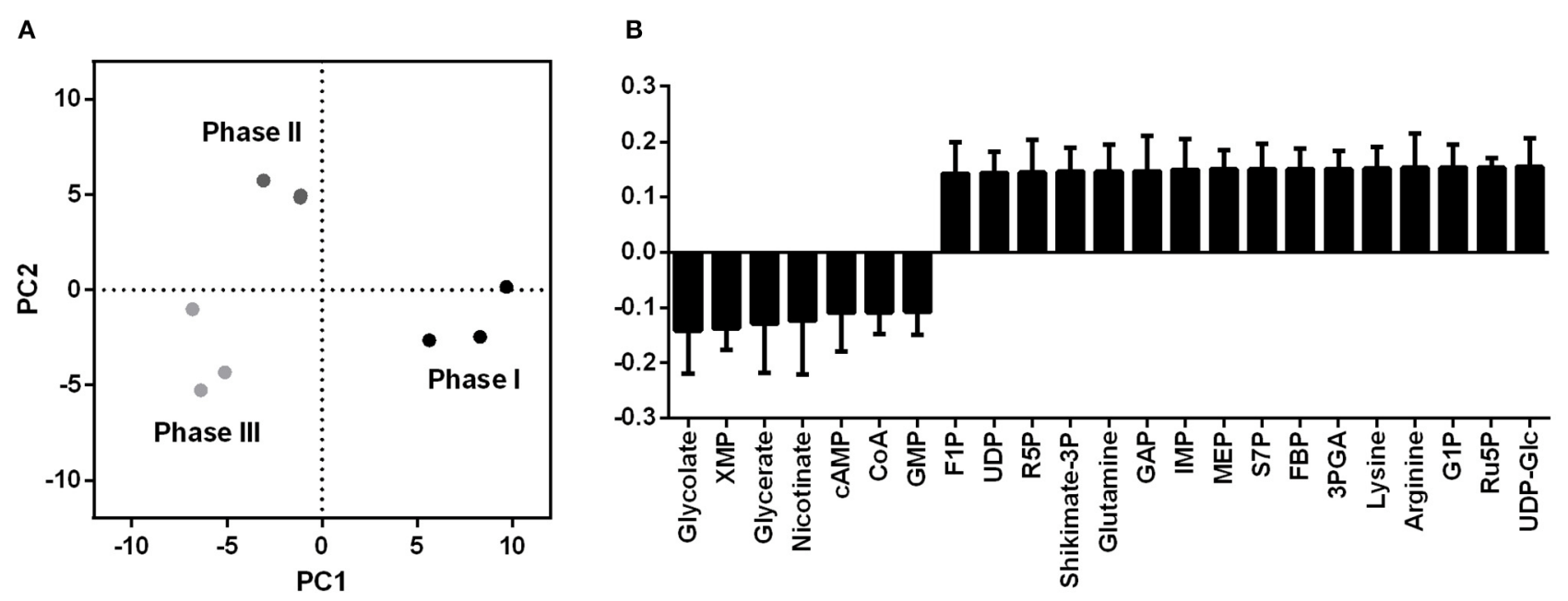

FIGURE 5 | Principal component analysis (PCA) for different growth phases of B. subtilis WB PX43-pgs on xylose. The score plot (A) demonstrates the separation according to principal component 1 (PC1) and PC2 for triplicate samples obtained from three cultivation time points corresponding to the phases indicated in Figure 4. Samples were taken after 9,11 , and $14 \mathrm{~h}$ for phase I, II, and III, respectively. The loading data for metabolites exhibiting the highest values for PC1, thus changing throughout the growth phases, are shown in (B). 3PGA, 3-phosphoglycerate; AMP, adenosine 5'-monophosphate; cAMP, 3',5'-cyclic AMP; CoA, coenzyme A; F1P, fructose-1-phosphate; FBP, fructose-bisphosphate; G1P, glucose-1-phosphate; GAP, D-glyceraldehyde 3-phosphate; GMP, guanosine 5'-monophosphate; IMP, inosine 5'-monophosphate; MEP, methyerythritol 4-phosphate; R5P, ribose-5-phosphate; Ru5P, ribulose-5-phosphate; S7P, sedoheptulose-7-phosphate; UDP, uridine 5'-diphosphate; UDP-Glc, uridine 5'-diphosphate-glucose; XMP, xanthosine 5'-phosphate. 


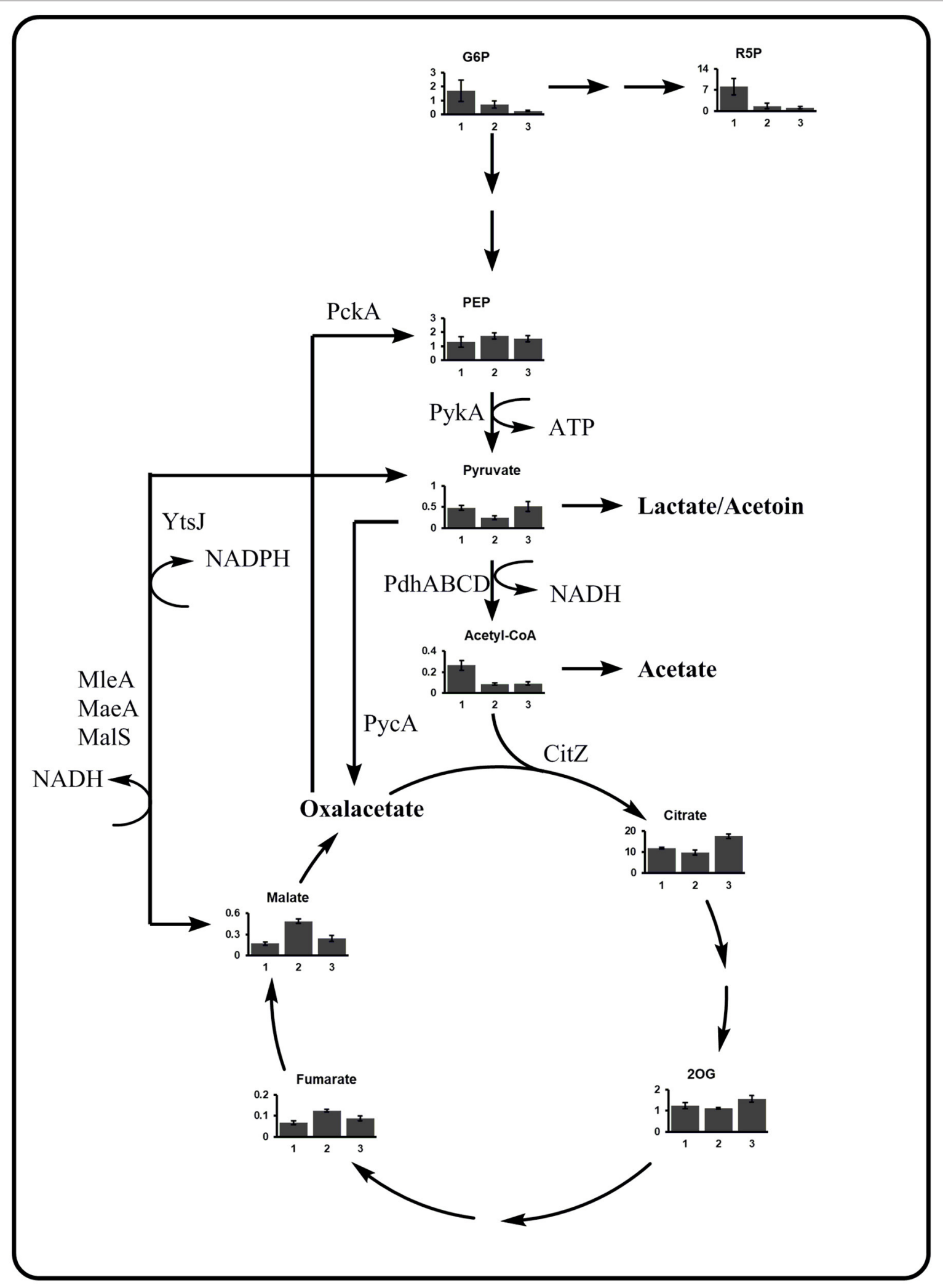

FIGURE 6 | Metabolic profile of the PEP-pyruvate-oxaloacetate node for B. subtilis WB growing on xylose. The metabolite concentrations from the central carbon metabolism for the three growth phases as indicated in 4 are given. Samples were taken after 9, 11, and $14 \mathrm{~h}$ for phase I, II, and III, respectively. Cultivations were 
FIGURE 6 | carried out in triplicates. The bar graphs represent the mean. The error bar indicates the standard deviation. CitZ, citrate synthase; MaeA, malic enzyme; MalS, malic enzyme; Mdh, malate dehydrogenase; MleA, malic enzyme; PdhABCD, pyruvate dehydrogenase; PckA, phosphoenolpyruvate carboxykinase; PPP, pentose phosphate pathway; PycA, pyruvate carboxylase; PykA, pyruvate kinase; YtsJ, malic enzyme (modified from Meyer and Stülke, 2013). 20G, 2-oxoglutarate; DHAP, dihydroxyacetone phosphate; F6P, fructose-6-phosphate; FBP, fructose-bisphosphate; G6P, glucose-6-phosphate; GAP, D-glyceraldehyde 3-phosphate; R5P, ribose-5-phosphate; S7P, sedoheptulose-7-phosphate.

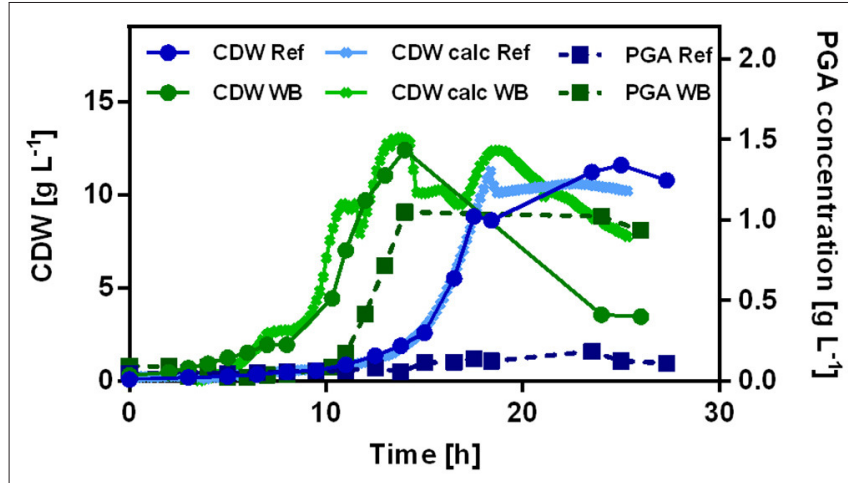

FIGURE 7 | Batch fermentation of B. subtilis Ref PX43-pgs (blue) and B. subtilis WB PX43-pgs (green) on xylose. The cell dry weight was monitored offline and online using the BioR-CGQ (circles). The $\gamma$-PGA concentrations are depicted (squares).

calculation of optimal $\gamma$-PGA production rates as basis for targeted strain and process development.

The theoretical flux distributions for the optimization of $\gamma$ PGA synthesis rate under varying substrate conditions are shown in Figure 8. The growth on glucose as sole carbon source (1), xylose via the xylose isomerase pathway (2), using the Weimberg pathway (4), or both (3) and a calculated theoretical optimal mixture of glucose and xylose (5) were modeled. Glucose as carbon source results in the highest theoretical synthesis rate. Here, the substrate is mainly converted to glutamate employing glycolysis and the TCA cycle resulting in a theoretical glutamate yield of $99 \%$ of the substrate uptake. In this scenario, only $1 \%$ of the carbon flux is directed to the PPP at the glucose-6P branch point.

The metabolism of xylose as sole carbon source for growth and $\gamma$-PGA production was tested by two pathways, isomerase pathway and Weimberg pathway. When only the Weimberg pathway is present (4), xylose is completely converted to 2oxoglutarate. For the supply of biomass precursors, 2-OG has to be partly converted to oxaloacetate that is subsequently used for gluconeogenesis via anaplerotic reactions (malic enzyme and PEP carboxykinase). For the given growth and ATP maintenance parameters, the theoretical flux distribution for the utilization of the PPP (2) is beneficial in terms of maximizing the $\gamma$ PGA production rate. The higher theoretical $\gamma$-PGA production rate for scenario (2) compared to (4) is likely due to precursor supply for biomass formation. Xylose metabolism via the Weimberg pathway requires energy-consuming gluconeogenesis. This catabolic pathway releases more carbon in form of $\mathrm{CO}_{2}$ since the Weimberg pathway includes the conversion 2-oxoglutarate to oxaloacetate which is further converted to phosphoenolpyruvate. In both reactions, $\mathrm{CO}_{2}$ is formed.

The theoretical $\gamma$-PGA production rate from xylose as sole carbon source is the highest when both xylose utilization pathways are present in the cells (3). In that case, ideally 30\% of xylose are converted to R5P using the isomerase pathway. The remaining xylose is metabolized via the Weimberg pathway resulting in a theoretical glutamate synthesis rate that is $88 \%$ of the substrate uptake rate. An even higher theoretical synthesis rate is achieved when a mixture of glucose and xylose is used (5). For this flux distribution, all xylose that is taken up is converted to glutamate via the Weimberg pathway. Precursors for biomass formation and redox equivalents are produced from glucose. Thereby a theoretical glutamate synthesis rate of $93 \%$ is calculated. While the synthesis rate is a bit lower as for glucose as carbon source, the theoretical carbon yield is higher for the substrate mix. However, the model does not include the consecutive uptake of the two substrates.

Modeling the flux distributions for $\gamma$-PGA-producing $B$. subtilis, the model revealed that the Weimberg pathway for xylose utilization is superior to the native xylose isomerase pathway when mixtures of glucose and xylose are used as substrate. Based on the given metabolic model, the optimized $\gamma$-PGA production rate and the theoretical yield were calculated as a function of substrate composition by FBA. The substrate was a mixture of glucose and xylose. The variation in the portions of glucose and xylose was modeled (Figure 9). When using a fixed growth rate, ATP maintenance rate and overall substrate uptake rate, the $\gamma$-PGA production rate increases with an increase in the glucose portion. However, the theoretical C-mol yield exhibits an optimum for a substrate mixture with $30 \%$ glucose. The absolute value for the theoretical yield varies between 0.78 and $0.88 \mathrm{C}-\mathrm{mol}$ $\gamma$-PGA/C-mol substrate. While the formation of biomass and ATP is more efficient using glucose via glycolysis and TCA as substrate, the availability of xylose via the Weimberg pathway is beneficial for maximizing the theoretical $\gamma$-PGA yield since xylose is converted to glutamate without carbon loss in form of $\mathrm{CO}_{2}$.

\section{$\gamma$-PGA Production Under Varying Substrate Concentrations}

For growth of B. subtilis WB PX43-pgs on a substrate mix of glucose and xylose, glucose is the preferred substrate. The growth curves on a mixture of glucose and xylose demonstrate a short lag phase indicating the shift from glucose to xylose metabolism that occurs later the higher the portion of glucose was chosen (Figure 10). Moreover, the growth curves emphasize that the growth rate of $B$. subtilis WB PX43-pgs on glucose is higher than on xylose ( 0.6 and $0.4 \mathrm{~h}^{-1}$, respectively). 


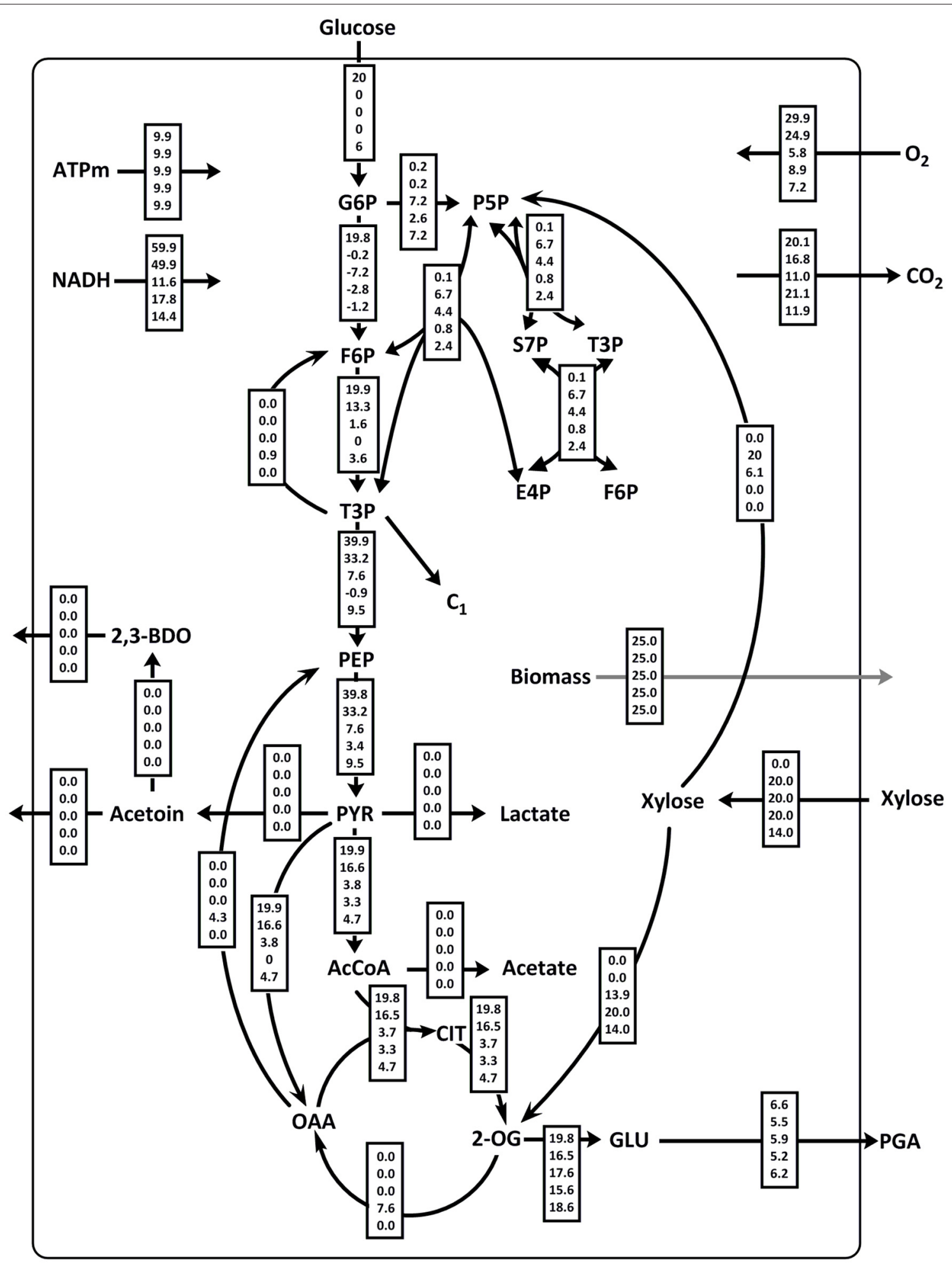

FIGURE 8 | Theoretical flux distributions for $\gamma$-PGA producing $B$. subtilis. Flux balance analysis (FBA) was used to calculate the fluxes for growth on glucose (1), xylose via isomerase pathway (2), xylose via isomerase and Weimberg pathway (3), xylose via Weimberg pathway (4), and a glucose/xylose mixture (5).

The effect of substrate ratio on the metabolism was also investigated by metabolome analysis. Intracellular metabolite extractions were carried out after $9 \mathrm{~h}$ at optical densities ranging between 8 for higher glucose concentrations and 6 for lower glucose concentrations. Differences in the intracellular metabolites caused by the substrate variation were evaluated by 


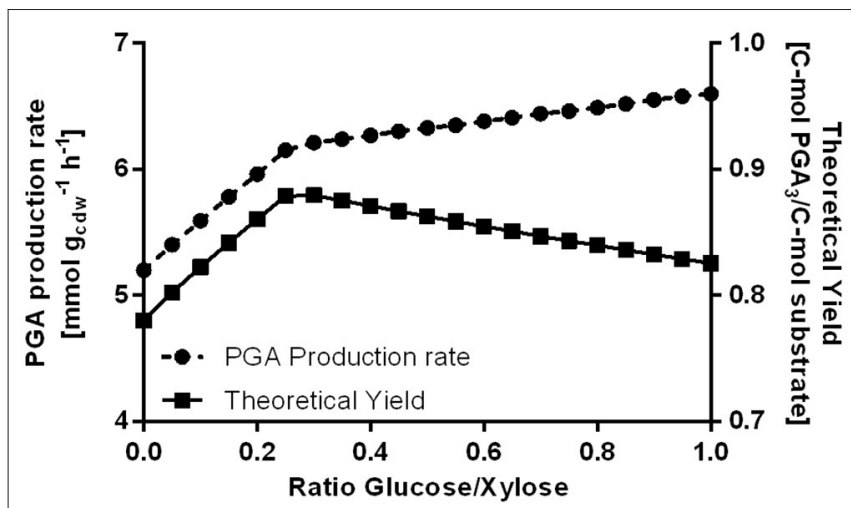

FIGURE 9 | Modeling the $\gamma$-PGA production rate and the theoretical yield for varying substrate ratios. The total substrate uptake rate was kept constant at $20 \mathrm{mmol} \mathrm{g}_{\mathrm{cdw}}^{-1} \mathrm{~h}^{-1}$. The biomass formation rate was set to $25 \mathrm{mmol} \mathrm{g}_{\mathrm{cdw}}^{-1} \mathrm{~h}^{-1}$ and the ATP maintenance coefficient was $9.9 \mathrm{mmol} \mathrm{g}_{\mathrm{cdw}}^{-1} \mathrm{~h}^{-1}$.

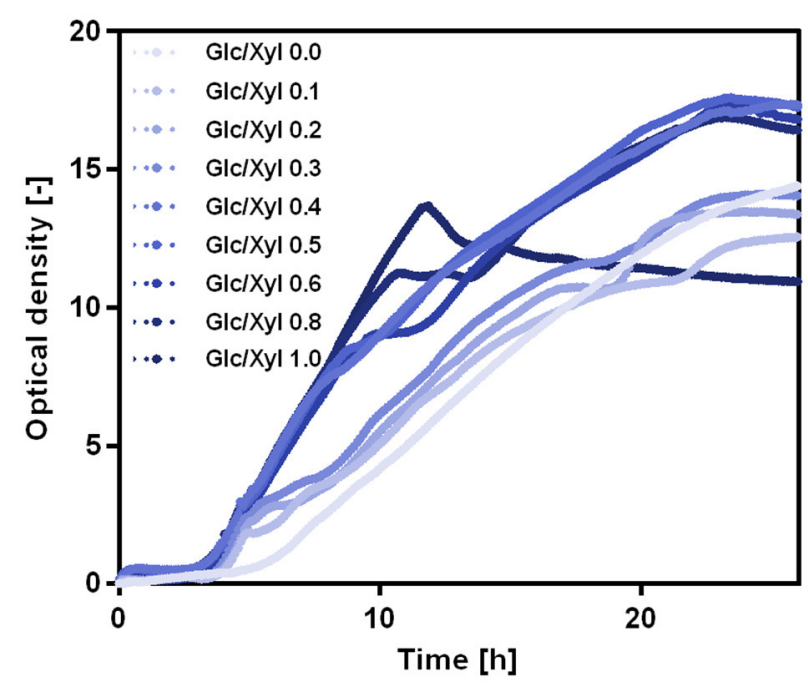

FIGURE 10 | Growth curves for B. subtilis WB PX43-pgs with varying substrate ratios. The total substrate of $20 \mathrm{~g} \mathrm{~L}^{-1}$ was varied from 0 to $100 \%$ glucose (light blue to dark blue). The cultivations were carried out in $500 \mathrm{~mL}$ shake flasks with $50 \mathrm{~mL}$ filling volume and $200 \mathrm{rpm}$ shaking frequency. The growth curves were monitored online using the CGQ (Aquila Biolabs). The data represent the mean of triplicate cultivations.

PCA (Figure 11). The first component is mainly representing the energy status of the cells, showing increased values for ATP, NADP, and NAD. The cAMP concentration decreased with PC1. The presence of cAMP in B. subtilis was detected for oxygen limited conditions (Mach et al., 1984). However, the role of cAMP is not comparable to the role in other bacteria such as E. coli where it is involved in altered gene expression as a second messenger controlling substrate uptake, motility or virulence (Makman and Sunderland, 1965; de Crombrugghe et al., 1984; Kalia et al., 2013). Besides energy-metabolism related metabolites, several TCA cycle intermediates (citrate, 2OG, and succinate) are increased for increasing PC1 values.
These findings agree with the results for the comparison of the Weimberg pathway and the xylose isomerase pathway. As stated before, the conversion of xylose via the Weimberg pathway likely causes a higher TCA cycle flux. As a result, the concentrations of TCA cycle intermediates decrease with higher xylose concentrations. The loading data for PC2 indicate increasing lactate concentrations for higher glucose concentration likely caused by induced overflow metabolism (Nakano et al., 1997; Cruz Ramos et al., 2000; Sonenshein, 2007). Moreover, some amino acids that are derived from glycolysis intermediates (leucine, valine) exhibit higher concentrations for higher glucose ratios. With higher xylose ratios several intermediates of purine metabolism correlate positively. Furthermore, some PPP metabolites such as RuBP, SBP, S7P, and the therefrom derived amino acid histidine exhibit increasing concentrations. Higher concentrations for intermediates from PPP have also been found when comparing the Weimberg pathway to the xylose isomerase pathway.

The $\gamma$-PGA production of $B$. subtilis Ref PX43-pgs (Figures 12A,B) and B. subtilis WB PX43-pgs (Figures 12C,D) was investigated for several substrate ratios including $0,20,40$, 60,80 , and $100 \%$ xylose. The $\gamma$-PGA production was monitored by on-line viscosity measurements (Sieben et al., 2019) and off-line $\gamma$-PGA concentration determination using the CTAB assay. Growth was closely monitored by measuring the OTR. Regestein et al. have shown that the $\gamma$-PGA concentration is closely related to viscosity (Regestein Née Meissner et al., 2017).

The OTR increases simultaneously during the first $7.5 \mathrm{~h}$ for both strains and all substrate ratios except for 100\% xylose (Figure 12A for reference and $\mathrm{C}$ for Weimberg mutant). Since glucose is the preferred substrate and the metabolic conversion of glucose is the same for both strains, no differences are observed until this time point. After that, the OTR drops for $80 \%$ xylose for both strains. For this glucose ratio, B. subtilis Ref PX43-pgs grows for $34 \mathrm{~h}$. After $34 \mathrm{~h}$, the OTR drops below $5 \mathrm{mmol} \mathrm{L}^{-1} \mathrm{~h}^{-1}$, which is slower compared to the Weimberg mutant. These results are in concordance with the lower growth rate of $B$. subtilis Ref PX43-pgs on xylose (Table 2 and Figure 7). Independent of the substrate composition, three maxima are observed for the OTR of B. subtilis Ref PX43-pgs (Figure 12A). These maxima correspond to the growth on glucose, on by-products such as 2,3-butanediol, and on xylose. For $20 \%$ xylose, a plateau at $40 \mathrm{mmol} \mathrm{L}^{-1} \mathrm{~h}^{-1}$ indicates an oxygen limitation. The determination of $\gamma$-PGA production by online viscosity measurements shows an increase of viscosity from $7 \mathrm{~h}$ on for all substrate compositions except 100\% xylose (reference) (Figure 12B). The maximal viscosity strongly depends on the substrate composition reaching a higher viscosity the more glucose is present. The highest viscosity obtained for B. subtilis Ref PX43-pgs was $14 \mathrm{mPa}$ s for $100 \%$ glucose. This viscosity corresponds to $4.7 \mathrm{~g} \mathrm{~L}^{-1} \gamma$-PGA as determined by CTAB assay. The OTR curve for B. subtilis Ref PX43-pgs on $100 \%$ xylose increases only after $10 \mathrm{~h}$ indicating a long lag phase for growth on xylose via the isomerase pathway. $\gamma$-PGA was poorly observed for the reference strain under these conditions.

For B. subtilis WB PX43-pgs, the OTR increased simultaneously to $22 \mathrm{mmol} \mathrm{L}^{-1} \mathrm{~h}^{-1}$ for all substrate ratios 

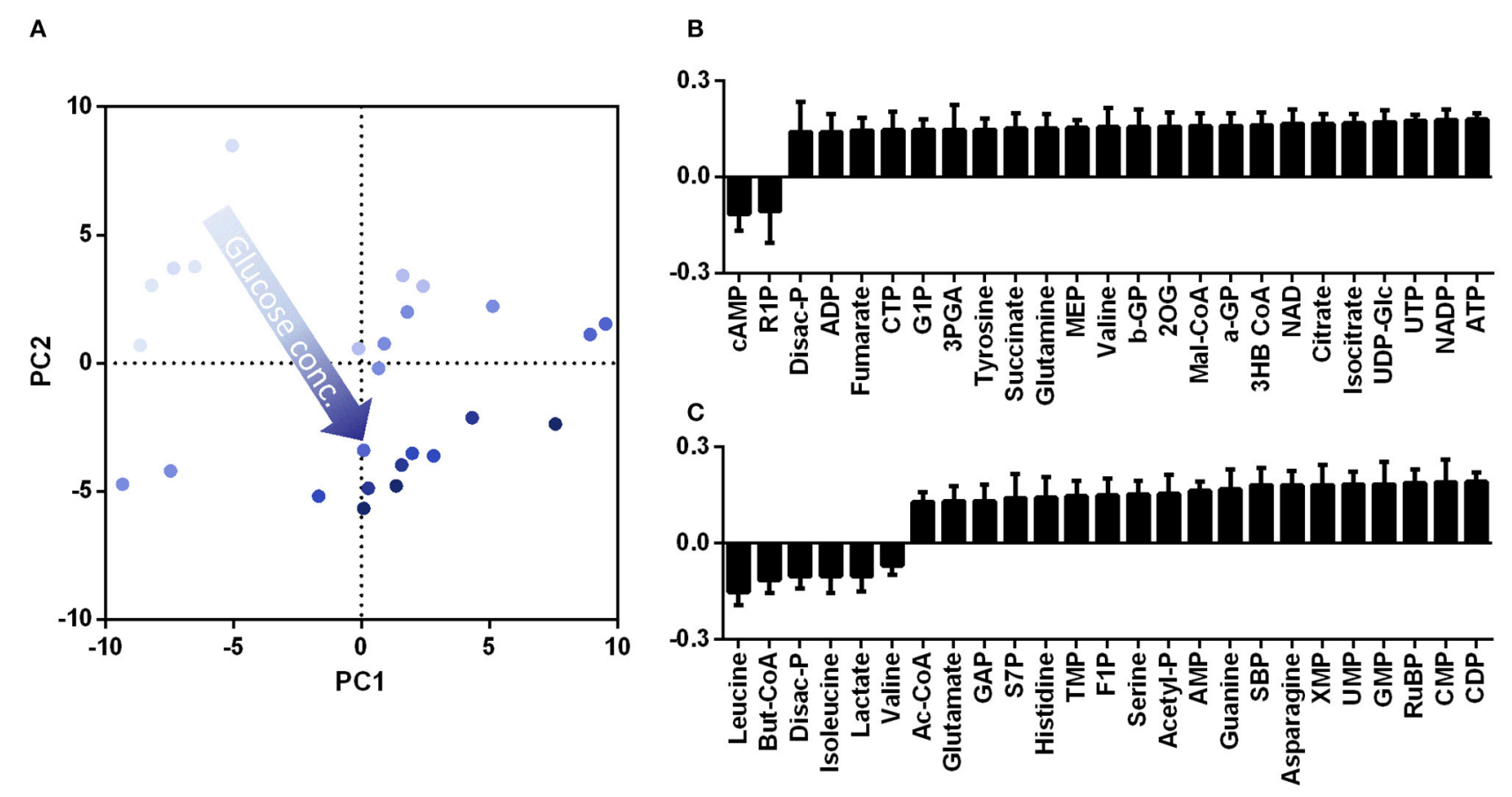

FIGURE 11 | Principal component analysis for substrate variations in B. subtilis WB PX43-pgs cultivations. The score plot (A) and loading data for PC1 (B) and PC2 (C) are given. The total substrate of $20 \mathrm{~g} \mathrm{~L}^{-1}$ was varied from 0 to $100 \%$ glucose (light blue to dark blue). The loading data for PC1 and PC2 is given for the metabolites with the highest impact on the principal components.

except $100 \%$ xylose during the first $7.5 \mathrm{~h}$ (Figure 12C). At this time point, the OTR for the cultures with the lowest glucose portion drops to $10 \mathrm{mmol} \mathrm{L}^{-1} \mathrm{~h}^{-1}$, indicating the depletion of glucose. This decline in OTR was observed for the other cultures after $8.5,9$, and $9.4 \mathrm{~h}$ for 40,60 , and $80 \%$ glucose, respectively. In all cultures, the OTR starts increasing again approximately $0.5 \mathrm{~h}$ after the drop indicating the shift from glucose metabolism to xylose metabolism. For glucose ratios of $0.2,0.4$, and 0.6 , the OTR is constant at $40 \mathrm{mmol} \mathrm{L} \mathrm{L}^{-1} \mathrm{~h}^{-1}$ between 10.5 and 13.5 h. The constant OTR indicates an oxygen limitation. After $13.5 \mathrm{~h}$, the OTR started to decline and reached $\sim 0 \mathrm{mmol} \mathrm{L}^{-1}$ $\mathrm{h}^{-1}$ after $35 \mathrm{~h}$. For the two highest glucose portions, a second OTR maximum after 13 and $14.5 \mathrm{~h}$ for 100 and $80 \%$ glucose, respectively, is detected, indicating the growth on a previously produced overflow metabolite. After that maximum, the OTR is constant at $25 \mathrm{mmol} \mathrm{L}^{-1} \mathrm{~h}^{-1}$ between 15.5 and $24 \mathrm{~h}$. After $24 \mathrm{~h}$, the OTR drops and reaches $0 \mathrm{mmol} \mathrm{L}^{-1} \mathrm{~h}^{-1}$ after $35 \mathrm{~h}$. In accordance with the lower growth rate of B. subtilis WB PX43-pgs on xylose, the OTR increases slower for $100 \%$ xylose reaching the maximum after $15 \mathrm{~h}$.

The $\gamma$-PGA production of $B$. subtilis WB PX43-pgs was monitored by online viscosity measurement (Figure 12D). The highest viscosity of $43 \mathrm{mPa} \cdot \mathrm{s}$ was observed for the culture with xylose as sole carbon source. With increasing glucose ratios, the maximal viscosity decreased to $30,22,19$, and $19 \mathrm{mPa} \cdot \mathrm{s}$ for 20 , 40,60 , and $80 \%$ glucose, respectively. After substrate depletion, the viscosity slightly decreased indicating the degradation of the produced $\gamma$-PGA. Besides the online viscosity measurement, the $\gamma$-PGA concentration was determined using the CTAB assay, with the same result: the maximal $\gamma$-PGA concentration of $5 \mathrm{~g} \mathrm{~L}^{-1}$ was obtained with $80 \%$ xylose. The produced $\gamma$-PGA corresponds to a $\mathrm{C}$-mol yield of 0.26 . With decreasing xylose portion, the $\gamma$-PGA titer decreased gradually with $4.5,4$, and $3.5 \mathrm{~g} \mathrm{~L}^{-1}$ for 60,40 , and $20 \%$ xylose, respectively.

In contrast to the Weimberg mutant, the $\gamma$-PGA production for B. subtilis Ref PX43-pgs was higher with higher glucose ratios (Figure 12E). Moreover, also the maximal viscosity was increased from $19 \mathrm{mPa} \cdot \mathrm{s}$ for the reference to $30 \mathrm{mPa} \cdot \mathrm{s}$ for the Weimberg mutant. Therefore, for the production of $\gamma$-PGA, the utilization of the Weimberg pathway is clearly superior to the xylose isomerase pathway.

\section{DISCUSSION}

Integration of the C. crescentus $x y l X A B C D$ genes was demonstrated to enable bacteria such as Pseudomonas (Meijnen et al., 2009) and Corynebacterium glutamicum (Radek et al., 2014) to grow on xylose as sole carbon and energy source. The direct conversion of xylose to the C5-compound 2-oxoglutarate without carbon loss is beneficial for $\gamma$-PGA production as well as for amino acids derived from 2-oxoglutarate. However, the reported maximal growth rate with $\mu=0.21 \mathrm{~h}^{-1}$ for $P$. putida S12 (Meijnen et al., 2009) and $\mu=0.07 \mathrm{~h}^{-1}$ for C. glutamicum (Radek et al., 2014) were low. In both cases, plasmid-based expression of the enzymes of the Weimberg pathway was used. The genomic integration of $x y l X A B C D$ in $B$. subtilis WB led to a growth rate of $0.43 \mathrm{~h}^{-1}$ (Table 2). The genomic integration circumvents the metabolic burden of plasmid replication. The 
A

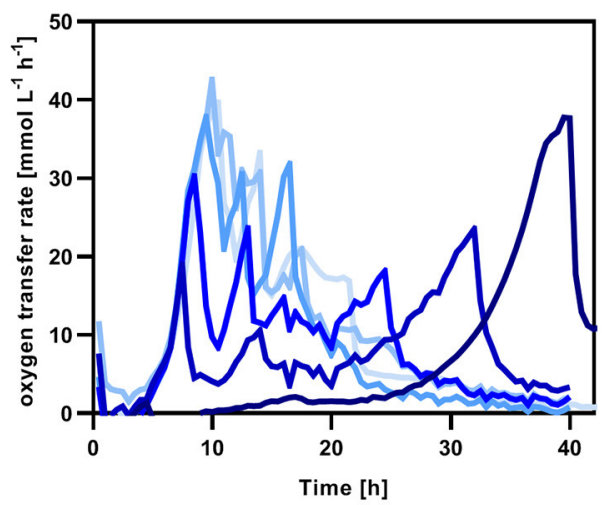

B

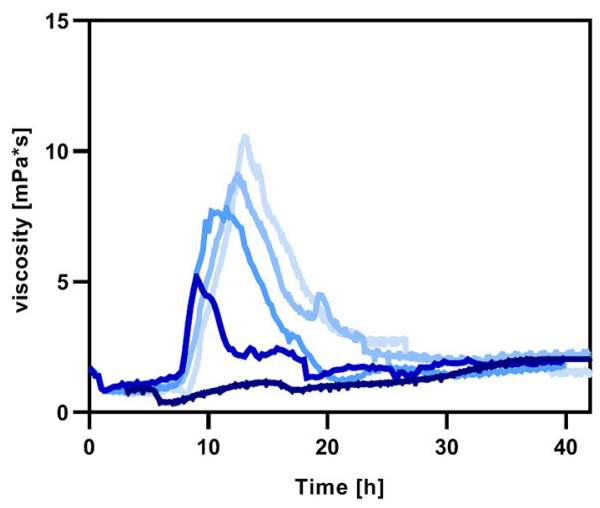

C

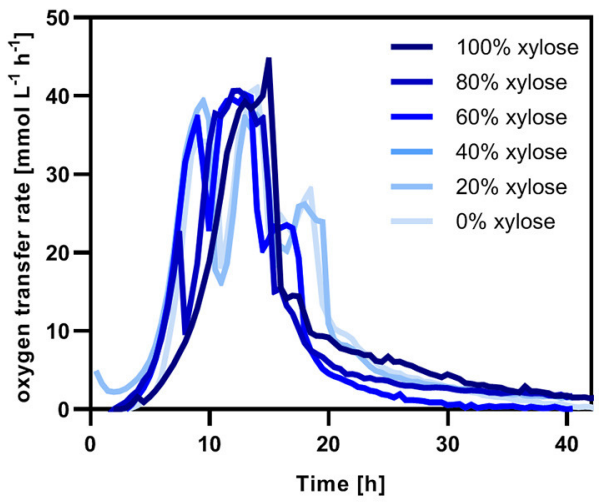

D

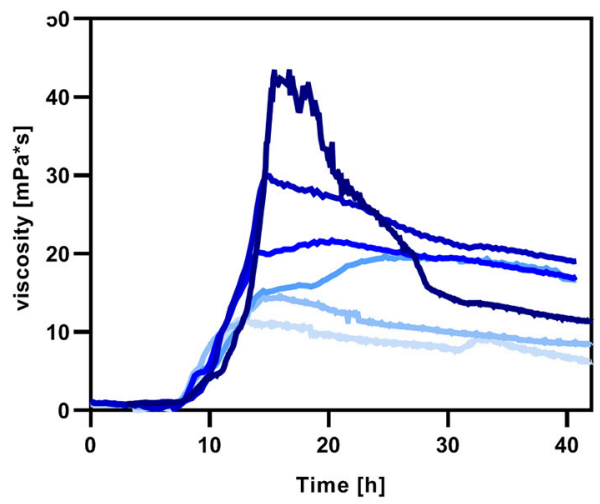

E

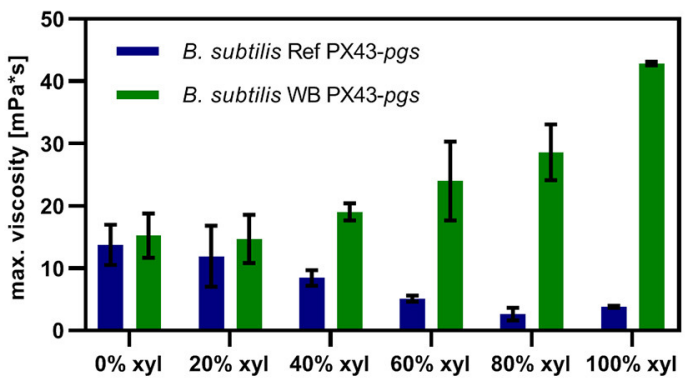

FIGURE 12 | Growth and $\gamma$-PGA production of engineered B. subtilis Ref PX43-pgs (reference; A,B) and B. subtilis WB PX43-pgs (C,D) in glucose/xylose minimal medium in shake flask cultivations. The oxygen transfer rate (OTR) representing the cell growth and metabolic activity of the cultured microorganisms is shown in (A) for the reference strain and in (C) for the Weimberg mutant. The $\gamma$-PGA measured as online viscosity is shown in (B) for B. subtilis Ref PX43-pgs and in (D) for $B$. subtilis WB PX43-pgs with glucose/xylose ratios varying from $0 \%$ xyl (100\% glucose, $0 \%$ xylose) to $100 \%$ xyl ( $0 \%$ glucose, $100 \%$ xylose). The total substrate concentration was kept constant at $20 \mathrm{~g} \mathrm{~L}^{-1}$ corresponding to $0.67 \mathrm{C}-\mathrm{mol} \mathrm{L}^{-1}$. The cultivations were carried out in triplicates for $20,40,60$, and $80 \%$ xylose and in duplicates for 0 and $100 \%$ xylose. The obtained maximal viscosity for the reference (blue) and Weimberg mutant (green) is given in (E).

replacement of the native $B$. subtilis xylose operon allowed for the regulation of expression by the native $B$. subtilis xylose promoter. The resulting growth rate was even higher than for the wild-type B. subtilis using the isomerase pathway.

In P. putida and C. glutamicum, the accumulation of xylonate was observed in the supernatant. Xylonate accumulation strongly indicates that the xylonate dehydratase reaction converting xylonate to 2-keto-3-desoxy-xylonate is the rate-limiting step of the pathway. The inefficient conversion of xylonate is likely one factor for the reduced growth rates in these bacteria. For
B. subtilis WB, the higher growth rate indicates a sufficient activity of all enzymes. Nevertheless, a bi-phasic (diauxic) growth with a lag phase between the two growth phases was observed. Here, intracellular metabolite measurements revealed the accumulation of TCA intermediates such as malate and fumarate. This indicates that the conversion of malate and oxaloacetate to pyruvate and phosphoenolpyruvate are ratelimiting steps in B. subtilis WB.

The two xylose utilization pathways, xylose isomerase and Weimberg pathway were compared with regard to the precursor 
supply for $\gamma$-PGA production. In contrast to the hypothesized higher 2-oxoglutarate supply for the Weimberg mutant, the concentration of most TCA cycle intermediates was higher for the xylose isomerase pathway. The metabolite measurements indicate a higher activity TCA cycle when xylose is converted to 2-oxoglutarate. This results in lower metabolite concentrations for TCA cycle intermediates. These results are consistent with the metabolome analysis for $\gamma$-PGA-producing $B$. licheniformis (Mitsunaga et al., 2016). For higher fluxes toward $\gamma$-PGA when glycerol is used as carbon source, the precursor concentrations of citrate, isocitrate, and 2-oxoglutarate are significantly lower than for glucose with a lower $\gamma$-PGA production rate. The higher TCA cycle activity was demonstrated to result in higher $\gamma$-PGA synthesis for the Weimberg mutant.

Several studies demonstrated an efficient $\gamma$-PGA synthesis with glutamic acid as additional carbon source (Cromwick et al., 1996; Richard and Margaritis, 2003). The majority of these studies involve wild-type $\gamma$-PGA producers that require glutamate for $\gamma$ PGA synthesis. For glutamate-independent production of $\gamma$-PGA the media commonly contain citric acid to enable high $\gamma$-PGA titers (Kongklom et al., 2017). In all cases, the $\gamma$-PGA production is greatly increased when direct glutamate precursors are used as substrates. The direct conversion of xylose to 2-oxoglutarate implemented in this study presents an alternative solution for higher precursor supply from cheaper substrates. Since up to $25 \%$ of lignocellulosic biomass are made up from pentoses (Lee, 1997), their use is essential to efficiently produces bio-based products.

When glucose and L-glutamate were used as carbon sources, only $6-9 \%$ of the glutamate that was incorporated into $\gamma$ PGA was de novo synthesized from glucose (Yao et al., 2010). Therefore, an increase in the initial glucose concentration mainly resulted in higher biomass formation instead of higher $\gamma$-PGA synthesis. Hence, the utilization of two carbon sources may be beneficial if one carbon source is used for growth and energy production and another one is used for $\gamma$-PGA precursor supply. The investigation of the theoretical flux distributions emphasized this hypothesis. A higher theoretical $\gamma$-PGA production rate was observed for glucose and xylose mixtures compared to xylose alone. For substrate mixtures, glucose was converted to biomass precursors and used as energy source. Xylose was converted to glutamate without carbon loss. However, the consecutive uptake of the two substrates is not considered in the theoretical flux distribution. Further engineering may focus on the utilization of both carbon sources (Wu et al., 2016). In this study, the

\section{REFERENCES}

Anderlei, T., and Büchs, J. (2001). Device for sterile online measurement of the oxygen transfer rate in shaking flasks. Biochem. Eng. J. 7, 157-162. doi: 10.1016/S1369-703X(00)00116-9

Anderlei, T., Zang, W., Papaspyrou, M., and Büchs, J. (2004). Online respiration activity measurement (OTR, CTR, RQ) in shake flasks. Biochem. Eng. J. 17, 187-194. doi: 10.1016/S1369-703X(03) 00181-5

Antoniewicz, M. R. (2015). Methods and advances in metabolic flux analysis: a mini-review. J. Ind. Microbiol. Biotechnol. 42, 317-325. doi: $10.1007 / \mathrm{s} 10295-015-1585-\mathrm{x}$ integration of promoter PX43 for PGA synthetase aimed at the discrimination of growth phase on glucose and production phase on xylose. Since the viscosity started increasing before glucose depletion, the PGA synthetase was expressed despite of the cre site for glucose repression. Furthermore, the theoretical flux distributions strongly depend on the growth rate and substrate uptake rate. A growth rate of $0.6 \mathrm{~h}^{-1}$ as used for the FBA was only proven to be true for growth on glucose. The changing growth rate throughout the cultivation was not considered.

In this study, the Weimberg pathway was successfully implemented into B. subtilis to achieve high $\gamma$-PGA production from biomass-derived substrates. Thereby, the carbon loss for utilization of xylose as substrate was minimized. The use of a mixture of glucose and xylose enabled the $\gamma$-PGA synthesis with a yield of $0.26 \mathrm{C}-\mathrm{mol} / \mathrm{C}-\mathrm{mol}$. The yield may further be improved by strain and process development to achieve resource efficient $\gamma$-PGA production from biomass-derived substrates.

\section{DATA AVAILABILITY STATEMENT}

All datasets generated for this study are included in the article/Supplementary Material.

\section{AUTHOR CONTRIBUTIONS}

$\mathrm{BH}$ and $\mathrm{LB}$ conceived and designed the study. SP and EF contributed to the design and data analysis of metabolomics experiments. $\mathrm{BH}, \mathrm{KH}, \mathrm{RH}, \mathrm{LB}$, and JB contributed to the design and data analysis of online viscosity experiments. $\mathrm{KH}$ and $\mathrm{RH}$ performed the online viscosity measurements. $\mathrm{BH}$ performed the remaining experiments and drafted the manuscript. All authors revised the manuscript and approved the final manuscript.

\section{FUNDING}

This work was funded by the Deutsche Forschungsgemeinschaft (DFG) within the International Research Training Group 1628, Selectivity in Chemo- and Biocatalysis (SeleCa).

\section{SUPPLEMENTARY MATERIAL}

The Supplementary Material for this article can be found online at: $\quad$ https://www.frontiersin.org/articles/10.3389/fbioe.2019. 00476/full\#supplementary-material

Ashiuchi, M. (2010). Occurrence and Biosynthetic Mechanism of Poly-Gamma Glutamic Acid. Amino Acid Homopolymers Occurring in Nature (Microbiology Monographs 15). Berlin; Heidelberg: Springer.

Belitsky, B. R., Wray, L. V., Fisher, S. H., Bohannon, D. E., and Sonenshein, A. L. (2000). Role of TnrA in nitrogen source-dependent repression of Bacillus subtilis glutamate synthase gene expression. J. Bacteriol. 182, 5939-5947. doi: 10.1128/JB.182.21.5939-5947.2000

Bohannon, D. E., Rosenkrantz, M. S., and Sonenshein, A. L. (1985). Regulation of Bacillus subtilis glutamate synthase genes by the nitrogen source. J. Bacteriol. 163, 957-964.

Cromwick, A. M., Birrer, G. A., and Gross, R. A. (1996). Effects of pH and aeration on y-poly(glutamic acid) formation by Bacillus licheniformis in controlled batch 
fermentor cultures. Biotechnol. Bioeng. 50, 222-227. doi: 10.1002/(SICI)10970290(19960420)50:2<222::AID-BIT10>3.0.CO;2-P

Cruz Ramos, H., Hoffmann, T., Marino, M., Nedjari, H., Presecan-Siedel, E., Dreesen, O., et al. (2000). Fermentative metabolism of Bacillus subtilis: physiology and regulation of gene expression. J. Bacteriol. 182, 3072-3080. doi: $10.1128 / J B .182 .11 .3072-3080.2000$

Dauner, M., and Sauer, U. (2001). Stoichiometric growth model for riboflavin-producing Bacillus subtilis. Biotechnol. Bioeng. 76, 132-143. doi: 10.1002/bit.1153

de Crombrugghe, B., Busby, S., and Buc, H. (1984). Cyclic AMP receptor protein: role in transcription activation. Science 224, 831-838. doi: $10.1126 /$ science. 6372090

Fujita, Y., Ramaley, R., and Freese, E. (1977). Location and properties of glucose dehydrogenase in sporulating cells and spores of Bacillus subtilis. J. Bacteriol. $132,282-293$.

Halmschlag, B., Steurer, X., Putri, S. P., Fukusaki, E., and Blank, L. M. (2019). Tailor-made poly- $\gamma$-glutamic acid production. Metab. Eng. 55, 239-248. doi: 10.1016/j.ymben.2019.07.009

Harwood, C. R. (1990). Molecular Biological Methods for Bacillus. John Wiley \& Sons Ltd, Cutting, S. M.

Kalia, D., Merey, G., Nakayama, S., Zheng, Y., Zhou, J., Luo, Y., et al. (2013). Nucleotide, c-di-GMP, c-di-AMP, cGMP, cAMP, (p)ppGpp signaling in bacteria and implications in pathogenesis. Chem. Soc. Rev. 42, 305-341. doi: $10.1039 / \mathrm{C} 2 \mathrm{CS} 35206 \mathrm{~K}$

Köhler, K. A., Blank, L. M., Frick, O., and Schmid, A. (2015). D-Xylose assimilation via the Weimberg pathway by solvent-tolerant Pseudomonas taiwanensis VLB120. Environ. Microbiol. 17, 156-170. doi: 10.1111/1462-2920. 12537

Kongklom, N., Shi, Z., Chisti, Y., and Sirisansaneeyakul, S. (2017). Enhanced production of poly- $\gamma$-glutamic acid by Bacillus licheniformis TISTR 1010 with environmental controls. Appl. Biochem. Biotechnol. 182, 990-999. doi: 10.1007/s12010-016-2376-1

Kreyenschulte, D., Krull, R., and Margaritis, A. (2014). Recent advances in microbial biopolymer production and purification. Crit. Rev. Biotechnol. 34, 1-15. doi: 10.3109/07388551.2012.743501

Lee, J. (1997). Biological conversion of lignocellulosic biomass to ethanol. J. Biotechnol. 56, 1-24. doi: 10.1016/S0168-1656(97)00073-4

Mach, H., Hecker, M., and Mach F. (1984). Evidence for the presence of cyclic adenosine monophosphate in Bacillus subtilis. FEMS Microbiol. Lett. 22, 27-30. doi: 10.1111/j.1574-6968.1984.tb00348.x

Makman, R. S., and Sunderland, E. W. (1965). Adenosine 3', 5'-phosphate in Escherichia coli. J. Biol. Chem. 240, 1309-1314.

Marciniak, B. C., Pabijaniak, M., de Jong, A., Duhring, R., Seidel, G., Hillen, W., et al. (2012). High- and low-affinity cre boxes for CcpA binding in Bacillus subtilis revealed by genome-wide analysis. BMC Genom. 13:401. doi: 10.1186/1471-2164-13-401

Meijnen, J. P., de Winde, J. H., and Ruijssenaars, H. J. (2009). Establishment of oxidative D-xylose metabolism in Pseudomonas putida S12. Appl. Environ. Microbiol. 75, 2784-2791. doi: 10.1128/AEM.02713-08

Meselson, M., and Yuan, R. (1968). DNA restriction enzyme from E. coli. Nature 217, 1110-1114. doi: 10.1038/2171110a0

Messing, J., Crea, R., and Seeburg, P. H. (1981). A system for shotgun DNA sequencing. Nucleic Acids Res. 9, 309-321. doi: 10.1093/nar/9.2.309

Meyer, F. M., and Stülke, J. (2013). Malate metabolism in Bacillus subtilis: distinct roles for three classes of malate-oxidizing enzymes. FEMS Microbiol. Lett. 339, 17-22. doi: 10.1111/1574-6968.12041

Mitsunaga, H., Meissner, L., Palmen, T., Bamba, T., Büchs, J., and Fukusaki, E. (2016). Metabolome analysis reveals the effect of carbon catabolite control on the poly( $\gamma$-glutamic acid) biosynthesis of Bacillus licheniformis ATCC 9945. J. Biosci. Bioeng. 121, 413-419. doi: 10.1016/j.jbiosc.2015. 08.012

Nakano, M. M., Dailly, Y. P., Zuber, P., and Clark, D. P. (1997). Characterization of anaerobic fermentative growth of Bacillus subtilis: identification of fermentation end products and genes required for growth. J. Bacteriol. 179, 6749-6755. doi: 10.1128/jb.179.21.6749-6755.1997
Oh, Y. K., Palsson, B. O., Park, S. M., Schilling, C. H., and Mahadevan, R. (2007). Genome-scale reconstruction of metabolic network in Bacillus subtilis based on high-throughput phenotyping and gene essentiality data. J. Biol. Chem. 282, 28791-28799. doi: 10.1074/jbc.M703759200

Radek, A., Krumbach, K., Gätgens, J., Wendisch, V. F., Wiechert, W., Bott, M., et al. (2014). Engineering of Corynebacterium glutamicum for minimized carbon loss during utilization of D-xylose containing substrates. J. Biotechnol. 192(Pt A):156-160. doi: 10.1016/j.jbiotec.2014.09.026

Regestein Née Meissner, L., Arndt, J., Palmen, T. G., Jestel, T., Mitsunaga, H., Fukusaki, E., et al. (2017). Investigation of $\operatorname{poly}(\gamma$-glutamic acid) production via online determination of viscosity and oxygen transfer rate in shake flasks. $J$. Biol. Eng. 11:23. doi: 10.1186/s13036-017-0065-4

Richard, A., and Margaritis, A. (2003). Optimization of cell growth and poly(glutamic acid) production in batch fermentation by Bacillus subtilis. Biotechnol. Lett. 25, 465-468. doi: 10.1023/a:1022644417429

Saha, B. C. (2003). Hemicellulose bioconversion. J. Ind. Microbiol. Biotechnol. 30, 279-291. doi: 10.1007/s10295-003-0049-x

Schellenberger, J., Que, R., Fleming, R. M., Thiele, I., Orth, J. D., Feist, A. M., et al. (2011). Quantitative prediction of cellular metabolism with constraint-based models: the COBRA Toolbox v2.0. Nat. Protoc. 6, 1290-1307. doi: 10.1038/nprot.2011.308

Sieben, M., Hanke, R., and Büchs, J. (2019). Contact-free determination of viscosity in multiple parallel samples. Sci. Rep. 9:8335. doi: 10.1038/s41598-019-44859-z

Sonenshein, A. L. (2007). Control of key metabolic intersections in Bacillus subtilis. Nat. Rev. Microbiol. 5, 917-927. doi: 10.1038/nrmicrol772

Stephens, C., Christen, B., Fuchs, T., Sundaram, V., Watanabe, K., and Jenal, U. (2007). Genetic analysis of a novel pathway for D-xylose metabolism in Caulobacter crescentus. J. Bacteriol. 189, 2181-2185. doi: 10.1128/JB.01438-06

van Gulik, W. M., de Laat, W. T., Vinke, J. L., and Heijnen, J. J. (2000). Application of metabolic flux analysis for the identification of metabolic bottlenecks in the biosynthesis of penicillin-G. Biotechnol. Bioeng. 68, 602-618. doi: 10.1002/ (SICI) 1097-0290(20000620)68:6<602::AID-BIT3>3.0.CO;2-2

Weimberg, R. (1961). Pentose Oxidation by Pseudomonas fragi. J. Biol. Chem. 236, 629-635.

Wenzel, M., and Altenbuchner, J. (2015). Development of a markerless gene deletion system for Bacillus subtilis based on the mannose phosphoenolpyruvate-dependent phosphotransferase system. Microbiology 161, 1942-1949. doi: 10.1099/mic.0.000150

Wenzel, M., Müller, A., Siemann-Herzberg, M., and Altenbuchner, J. (2011). Selfinducible Bacillus subtilis expression system for reliable and inexpensive protein production by high-cell-density fermentation. Appl. Environ. Microbiol. 77, 6419-6425. doi: 10.1128/AEM.05219-11

Wu, G., Yan, Q., Jones, J. A., Tang, Y. J., Fong, S. S., and Koffas, M. A. G. (2016). Metabolic burden: cornerstones in synthetic biology and metabolic engineering applications. Trends Biotechnol. 34, 652-664. doi: 10.1016/j.tibtech.2016.02.010

Yao, J., Xu, H., Shi, N., Cao, X., Feng, X., Li, S., et al. (2010). Analysis of carbon metabolism and improvement of $\gamma$-polyglutamic acid production from Bacillus subtilis NX-2. Appl. Biochem. Biotechnol. 160, 2332-2341. doi: 10.1007/s12010-009-8798-2

Zhu, F., Cai, J., Wu, X., Huang, J., Huang, L., Zhu, J., et al. (2013). The main byproducts and metabolic flux profiling of $\gamma$-PGA-producing strain B. subtilis ZJU-7 under different $\mathrm{pH}$ values. J. Biotechnol. 164, 67-74. doi: $10.1016 /$ j.jbiotec.2012.12.009

Conflict of Interest: The authors declare that the research was conducted in the absence of any commercial or financial relationships that could be construed as a potential conflict of interest.

Copyright $\odot 2020$ Halmschlag, Hoffmann, Hanke, Putri, Fukusaki, Büchs and Blank. This is an open-access article distributed under the terms of the Creative Commons Attribution License (CC BY). The use, distribution or reproduction in other forums is permitted, provided the original author(s) and the copyright owner(s) are credited and that the original publication in this journal is cited, in accordance with accepted academic practice. No use, distribution or reproduction is permitted which does not comply with these terms. 